\title{
Polymeric solid-state dye lasers: Recent developments
}

\author{
A. Costela, ${ }^{* a}$ I. García-Moreno ${ }^{a}$ and R. Sastre ${ }^{b}$ \\ "Instituto de Química Física "Rocasolano", CSIC, Serrano 119, 28006 Madrid, Spain. \\ E-mail: acostela@iqfr.csic.es; Fax: +34915642431 \\ ${ }^{b}$ Instituto de Ciencia y Tecnología de Polímeros, CSIC, Juan de la Cierva 3, \\ 28006 Madrid, Spain
}

Received 7th July 2003, Accepted 12th September 2003
First published as an Advance Article on the web 2nd October 2003

An up-to-date overview of the results obtained by our group on the development of polymeric solid-state dye lasers based on dipyrromethene $\mathrm{BF}_{2}$ complexes is presented. It is shown that appropriate chemical modifications in the dye molecules can yield dyes that lase efficiently and with remarkable photostability when properly incorporated into adequate polymeric matrices. Our results compare favourably with those reported by other authors with the dyes incorporated into organic materials and open the way to the development of solid-state dye lasers competitive with their liquid counterparts.

\section{Introduction}

From the mid 1960s, dye lasers have been attractive sources of coherent tunable visible radiation because of their unique operational flexibility. ${ }^{1}$ Dye lasers can emit both in pulsed and continuous-wave forms, can be pumped with a wide variety of excitation sources, and exhibit an inherent ability to yield high pulse energies and high average powers. Hundreds of dyes have been demonstrated to lase measurably covering the spectral range from the ultraviolet to the near infrared. The introduction of wavelength-selective elements in the laser cavity allows narrow-linewidth operation and tunability, and the large gain bandwidth of these molecules makes possible the generation of ultrashort pulses. The versatile nature of these lasers has resulted in their applicability to a wide range of different fields, from basic science, such as physics and spectroscopy, to medicine and industry.

Although dyes have been demonstrated to lase in the solid, liquid, or gas phases, liquid solutions of dyes in suitable organic solvents have been the most frequently used laser media because the active medium can be obtained in high optical quality and a liquid solution can be cooled by simply using a flow system. Nevertheless, the use of liquid solutions entrails some difficulties, mainly related to the need of employing large volumes of organic solutions of dyes that are both toxic and expensive to use and dispose of. Continuous circulation of the solution requires pumps and the design of complex and bulky cells, which, together with the large dye/solvent reservoirs, increases the size and cost of these dye laser systems and has restricted their use outside the laboratory.

From the early days of development of dye lasers, attempts were made to overcome the problems posed by organic solvents by incorporating the dye molecules into solid matrices. ${ }^{2,3}$ A solid-state dye laser avoids the problems of toxicity and flammability, presents a low cost gain medium, and is compact and easy to operate and maintain. Although this approach was very attractive, the first results were not very encouraging, with low lasing efficiencies and fast dye photodegradation, and as a result the research on solid-state dye lasers remained near dormant for over two decades. It was not until the late 1980s that developments of new and improved host materials with higher laser-damage resistance gave a new impulse to the field. ${ }^{4-6}$ In recent years, the synthesis of new high-performance dyes and the implementation of new ways of incorporating the organic molecules into the solid matrix has resulted in significant advances towards the development of practical tunable solidstate dye lasers. ${ }^{7}$

The basic requirements imposed on a solid matrix to be used as a host for lasing dye molecules, are high optical quality with a low level of scattering and a wide range of transparency covering both pump and lasing wavelengths. In addition, use of solid matrices in dye laser applications requires both materials with a high damage threshold to laser radiation and good thermal and photochemical stabilities and a simple technology for doping those materials with different classes of organic dyes. Although over the years a number of materials have been tried as solid hosts for organic dyes, most of the recent work has been done using inorganic glasses and transparent polymers.

Inorganic glasses have in general better thermal properties (higher thermal conductivity and lower thermal expansion and thermal coefficient of refractive index, $\partial n / \partial T)^{8,9}$ and a higher damage threshold than polymers. ${ }^{10,11}$ The low-temperature sol-gel process allowed the incorporation of organic dyes into silica gel inorganic glasses, resulting in relative photostable materials with good laser efficiency. ${ }^{12,13}$ Nevertheless, new modified polymers have been obtained with threshold of damage by laser radiation as high as those of the inorganic materials, and with stabilities higher than those of the silica gels under the same experimental conditions. ${ }^{14,15}$ Additional advantages of polymers include better chemical compatibility with organic dyes, superior optical homogeneity, ${ }^{11,16}$ control over medium polarity and viscosity in a way similar to conventional solvents, and adaptability to inexpensive fabrication techniques which facilitate both miniaturization and the design of integrated optical systems. Not less important is the easiness with which the structure and chemical composition of polymers can be modified, which allows to introduce controlled changes to optimise their properties when used in a particular application. ${ }^{7,17}$

Stimulated by the above advantages of polymers, our group began in the early 1990s a research program to fully explore the potential of these materials as host matrices for lasing dyes. The vast possibilities in polymer synthesis were utilized to modify the characteristics of the materials in a controlled 
manner; new methods for the incorporation of the active dye molecules into the polymeric matrices were implemented, and the lasing properties of the resulting materials were systematically evaluated. In a first stage we focused our attention in matrices incorporating dyes of the rhodamine family. The rhodamine dyes, with emission in the yellow-red region of the spectrum, were known to give excellent laser results in liquid solution, so that they were an obvious first election in any attempt to develop a dye laser in the solid state. In much of this work the dye of choice was Rhodamine $6 \mathrm{G}(\mathrm{Rh} 6 \mathrm{G})$, because there were numerous studies on this dye incorporated into different materials, which would facilitate the evaluation of our results by comparison with those previously reported in the literature. Our approach evidenced to be very effective, and lasing efficiencies similar to those found in ethanolic solution with improved photochemical stability, as compared with that obtained in previous polymeric materials, were demonstrated for solid solutions of Rh6G in appropriate copolymer formulations. ${ }^{18}$ Further improvements in photostability were obtained when modified Rh6G molecules were copolymerized with methacrylic monomers. ${ }^{18}$

Another promising line of research has been the search for more efficient and stable laser dyes. Triplet-triplet absorption over the laser spectral region turns out to be one of the most important causes limiting the laser performance of organic dyes. As a result of the continuous effort to produce improved dyes for laser applications, a new class of laser dyes with reduced triplet-triplet absorption was synthesized by Boyer and co-workers during the late 1980 s and early $1990 \mathrm{~s}^{19-25}$ and some of them are now commercially available. These dyes are dipyrromethene $\mathrm{BF}_{2}$ complexes, with emission covering the spectral region from the green-yellow to the red, depending on the substituents on the chromophore, high fluorescence quantum yields and low triplet extinction coefficients over the laser action spectral region that in some cases are only one-fifth of their rhodamine counterparts. ${ }^{26}$ They are highly polar laser dyes with zwitterionic structure exhibiting good solubility in many solvents, including alcohols and methyl methacrylate. Many dyes of this dipyrromethene $\mathrm{BF}_{2}$ family are highly efficient laser dyes, with low intersystem crossing and low excited-state absorption coefficients. ${ }^{20,22-24}$ In particular, some of these complexes outperform in flashlamp-pumped, laserpumped and continuous-wave operation the most widely employed laser dye, Rh6G, considered as the benchmark in efficiency and photostability. ${ }^{20,24,26-28}$ On the side of the disadvantages, the presence of amine aromatic groups in their structure renders these dipyrromethene dyes vulnerable to photochemical reactions with oxygen, which makes them relatively unstable in air saturated solutions. ${ }^{29}$

The promising laser-action properties of the dipyrromethene $\cdot \mathrm{BF}_{2}$ complexes made them attractive candidates to be incorporated into solid hosts, and after 1993 successful laser oscillation of various pyrromethene dyes incorporated into different host materials has been reported. ${ }^{7,30,31}$ Most of this work was performed with inorganic and hybrid hosts, although the first reported results were obtained using a modified acrylic plastic as the host material. ${ }^{14}$ By the mid 1990s, our studies with rhodamine dyes had provided ample evidence of the virtues and great potentiality of the polymeric materials as solid hosts for lasing dyes. These studies showed that lasing efficiencies and photostability depend on the viscoelastic properties of the medium and demonstrated that for each dye there is an optimum copolymer formulation that results in the best matrix/dye combination. A next logical step was to extend our research to the new, high-performance pyrromethene dyes and probe their lasing properties when incorporated into appropriate polymers. Thus, over the last years we have performed a systematic study of the laser performance of selected pyrromethene dyes in polymeric matrices. We began by using commercial pyrromethene dyes and, after characterizing their photophysical and lasing properties in a variety of solvents, proceeded to incorporate them into carefully chosen polymeric formulations, to gather information on the polymer parameters and structure composition which optimized the laser operation. Next, we proceeded to synthesize new dipyrromethene $\mathrm{BF}_{2}$ complexes and demonstrated that with appropriate chemical modifications in the pyrromethene chromophore new dyes could be obtained which outperformed the commercially available laser dyes.

In this paper, we present an up-to-date overview of the results obtained by our group on the development of polymeric solid-state dye lasers based on dipyrromethene $\mathrm{BF}_{2}$ complexes. Comparison with the results reported by other authors with the dyes incorporated into organic materials outlines the state-of-the-art in the field.

This paper is organized in the following way. In section 2, the structures of the different dyes and polymeric hosts utilized in our studies are depicted and the methods of preparation of the different materials are considered. Section 3 deals with the photophysics of the different dyes both in liquid and solid solution. The lasing properties of the different materials are considered in detail in section 4 , and the results obtained are compared with previous results by other authors. In section 5, some final general remarks and conclusions are presented, and consideration is given to prospects for future developments.

\section{Materials}

\subsection{Dyes}

As indicated in the previous section, the highly-colored dipyrromethene $\mathrm{BF}_{2}$ dyes have attracted considerable attention because some of them fulfill the necessary photophysical conditions for efficient dye laser emission, such as fluorescence emission quantum yields in organic solvents close to unity, ${ }^{32,33}$ low triplet-triplet absorption over their lasing spectral region and high photostability. We have used in our studies both commercial and newly synthesized dipyrromethene $\mathrm{BF}_{2}$ complexes. The molecular structures of three of the most usual commercial pyrromethene dyes are shown in Scheme 1: 1,3,5,7,8-pentamethyl-2,6-diethylpyrromethene-difluoroborate (PM567), 1,3,5,7,8-pentamethyl-2,6-di-n-butyl pyrromethenedifluoroborate (PM580), and 1,3,5,7,8-pentamethyl-2,6-di-tbutylpyrromethene-difluoroborate (PM597).

We have synthesized ${ }^{34}$ a series of new dipyrromethene $\cdot \mathrm{BF}_{2}$ dyes with the same substituents as PM567 in positions 1 to 7 , but containing at position 8 a methacryloyloxy group separated from the chromophore by a linear aliphatic chain with 1 , 3, 5, 10 or 15 methylenes (PnMA, Scheme 2). These new dyes have been copolymerized with acrylic and methacrylic monomers (Scheme 3), yielding copolymers with the covalently bounded chromophore separated from the polymeric

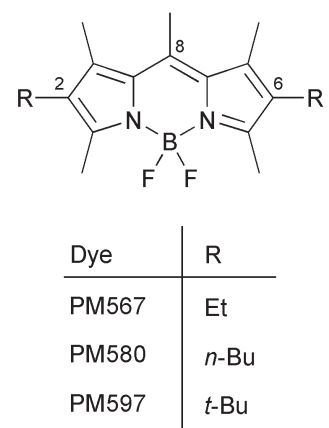

Scheme 1 Molecular structures of some commercial dipyrromethene $\cdot \mathrm{BF}_{2}$ complexes. $\mathrm{Bu}: \mathrm{CH}_{3}\left(\mathrm{CH}_{2}\right)_{3} ; \mathrm{Et}$ : $\mathrm{C}_{2} \mathrm{H}_{5}$. 


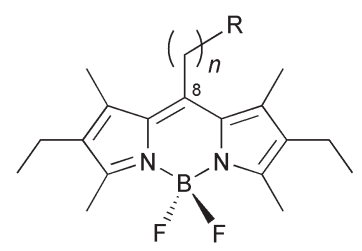

\begin{tabular}{l|ll} 
& $\mathrm{R}$ & $n$ \\
\hline PM567 & $\mathrm{H}$ & 1 \\
PnAc & OCOMe & $1,3,5,10,15$ \\
PnMA & OCOC $(\mathrm{Me})=\mathrm{CH}_{2}$ & $1,3,5,10,15$
\end{tabular}

Scheme 2 Molecular structures of newly synthesized modified dipyrromethene $\cdot \mathrm{BF}_{2}$ complexes. $\mathrm{Me}: \mathrm{CH}_{3}$.

backbone by an aliphatic chain of variable length. ${ }^{35}$ Our main aim was to try to correlate the separation between chromophore and polymeric main chain in these new materials with their photostability and emission properties. For comparison, we have also synthesized the corresponding model compounds with acetoxy group instead of the methacryloyloxy group(PnAc, Scheme 2), preparing with them solid polymeric materials where the dye is simply dissolved into polymer matrices.

\subsection{Polymer matrices}

The basic requirements imposed on a polymeric host for lasing dye molecules are good optical transparency at both pump and lasing wavelengths, good solubility of the dye in the material, and resistance to pump laser radiation. The acrylic and methacrylic polymers have been the most frequently used hosts for lasing dyes due to their excellent optical transparency in the visible and their relatively high laser-damage resistance. Nevertheless, some of the more usual dyes, such as Rh6G, have limited solubility in these materials, and the structural rigidity of these polymers could be restraining their resistance to laser damage. Although there is no general consensus concerning the mechanism responsible for laser damage of polymeric materials, there is already enough evidence to establish that the laser damage of polymers is energy dependent, and that the matrix viscoelastic properties determine the damage resistance. Thus, it would be expected that it would be possible to significantly improve the laser resistance of the material by changing the viscoelastic properties of the medium in the appropriate way. Following this line of reasoning, it had been demonstrated that the external plasticization of the polymer, by adding low molecular weight dopants, improves significantly the laser resistance. ${ }^{36}$

In our studies we tried a different but related approach, where the plasticity of the material was internally increased by copolymerization of different acrylic and methacrylic comonomers. The presence of particular comonomers not only can increase the plasticity of the material while maintaining the good transparency in the near-ultraviolet and visible

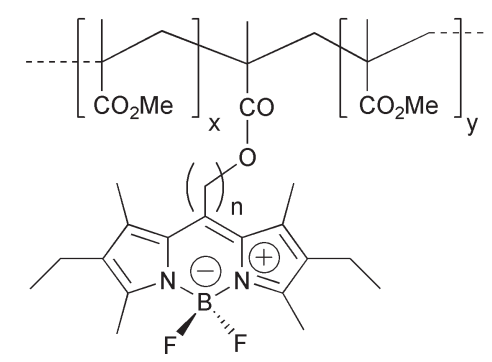

Scheme 3 Model structure of copolymers COP(PnMA/MMA). Me: $\mathrm{CH}_{3}$. MMA: methyl methacrylate. spectral ranges, but also insures a good solubility of the chosen dyes.

Our experience with polymeric matrices incorporating laser dyes indicates that the lasing properties of a dye-polymer material depend largely on the methods used for their manufacture. These methods are constrained by the stringent requirements placed upon the laser materials, which make the well-developed techniques for moulding polymeric materials not very well suited to ensure the high optical uniformity, absence of internal stress and purity of the final material needed in this application. An alternative to the more conventional methods uses the uniform distribution of a liquid polymer plus dye solution in an organic solvent over the appropriate optical surface, followed by the subsequent removal by evaporation of the solvent. A first choice involves bulk radical polymerization of the initial monomer/fluorophore composition and subsequent machining and polishing of the material so obtained. This is the method we have used to prepare our materials.

Although each particular dye-monomer system requires the optimization of some specific polymerization conditions, it must be emphasized the need of a careful purification of both dye and monomer, the appropriate filtration or ultrafiltration of the resulting solution to be polymerized, and its $\mathrm{O}_{2}$-degassing in order to avoid the inhibition of the radical polymerization by atmospheric oxygen. Another important aspect of the polymerization process is the choice of an initiator and its concentration, that should affect neither the dye molecules nor the optical properties of the resulting material.

In the preparation of solid polymeric samples each dye was dissolved with sonication in freshly purified monomer or monomers mixture $(10 \mathrm{~mL})$, adjusting the dye concentration so that the absorbance versus air of the final solid polymeric disks ( $1 \mathrm{~mm}$ thick, see latter) at the visible maximum, taking into account $c a$. $20 \%$ volume reduction after polymerization, was close to 2.0. This value corresponds to corrected absorbances at $310 \mathrm{~nm}$ (position of maximum emission of the UV light used in the photodegradation experiments) of 0.09-0.10 for the model dyes PnAc, and of 0.15-0.20 for the copolymers of the monomeric dyes PnMA. Corresponding absorbance of the parent dye in poly(methyl methacrylate) PM567/PMMA: 0.07 . The free radical polymerization initiator $2,2^{\prime}$-azobis (2methylpropionitrile) (AIBN) (Across Organics, crystallized from $\mathrm{MeOH}$ in the dark), a thermal initiator that leaves UVtransparent end-groups in the final polymer, was then added (30 $\mathrm{mg})$, and the mixture was again sonicated. The resulting solution was filtered into appropriate cylindrical (12 $\mathrm{mm}$ diameter) polypropylene molds through a $0.45 \mu \mathrm{m}$ pore filter and then through a $0.2 \mu \mathrm{m}$ pore filter (both Whatman Lab, polytetrafluoroethylene disposable filters). The filtered solution was deaerated with dry argon for $10 \mathrm{~min}$ and the mould was sealed. Polymerization was performed in the dark in a bath at $40^{\circ} \mathrm{C}$ until solidification (at least 2 days) and then at $45^{\circ} \mathrm{C}$ for 1 day. Next, the temperature was raised to $60^{\circ} \mathrm{C}$ and slowly increased to $80^{\circ} \mathrm{C}$ along 2 days in order to decompose residual AIBN. Finally, the temperature was reduced in steps of $5^{\circ} \mathrm{C}$ per day until room temperature was reached, and only then were the samples unmolded. The polymeric rods so prepared were cut in disks of $10 \mathrm{~mm}$ in diameter and $10 \mathrm{~mm}$ thick, and the flat surfaces of the disks were hand-polished until optical quality was reached, and used in the photodegradation studies.

The chromophore content in representative samples of copolymers was determined by dissolving each copolymer in the minimum volume of chloroform. The solution was slowly dropped into a high excess of vigorously stirred ethanol, and the precipitate was filtered off from the colorless solvent mixture and dried in vacuum $\left(10^{-4}\right.$ Torr, $\left.1.33 \times 10^{-2} \mathrm{~Pa}\right)$ until constant weight. The process was repeated, the final solid was dissolved in ethyl acetate, and the chromophore content 
was determined by absorption spectroscopy using the molar absorption coefficients found for the corresponding models $\mathrm{P} n \mathrm{Ac}$ and monomers PnMA in the same solvent.

The different dyes have been incorporated either in homopolymer PMMA or in a variety of copolymers of methyl methacrylate (MMA) with both monofunctional monomers (2-hydroxyethyl methacrylate (HEMA), 2-hydroxyethyl acrylate (HEA), 1-vinyl-2-pyrrolidone (VP), 2-phenoxyethyl acrylate (PEA), and 2,2,2-trifluoromethyl methacrylate (TFMA), Scheme 4, and cross-linking monomers (ethyleneglycol dimethacrylate (EGDMA), trimethylolpropane trimethacrylate (TMPTMA), tetraethyleneglycol diacrylate (TEGDA), pentaerythritol triacrylate (PETA), and pentaerythritol tetraacrylate (PETRA), Scheme 5.

\section{Photophysics}

\subsection{Experimental methods}

To study the photophysical and photochemical properties, disks $10 \mathrm{~mm}$ in diameter and $0.2 \mathrm{~mm}$ thick were cut out of the polymer samples and their flat surfaces hand polished until optical quality was obtained.

Absorption and fluorescence (after excitation at 490 or 510 $\mathrm{nm}$, depending on the dye) spectra were recorded on a Cary 4E spectrophotometer and on a Shimadzu RF-5000 spectrofluorimeter, respectively. Fluorescence quantum yields $(\phi)$ of PM567 and new dipyrromethene derivatives were determined using as reference a diluted rhodamine 110 solution in ethanol $\left(\phi=0.99\right.$ at $\left.20^{\circ} \mathrm{C}^{37}\right)$ and a solution of PM567 in methanol $\left(\phi=0.91^{27}\right)$, respectively. The $\phi$ values for PM567 doped solid samples were calculated considering a liquid solution as reference (a $2 \times 10^{-5} \mathrm{~mol} \mathrm{l}^{-1}$ solution of PM567 in ethanol, $1 \mathrm{~mm}$ cell), but the fluorescence intensity of the sample and the reference could not be registered in exactly the same way, owing to the different dispositions of the sample (solid) and the reference (liquid) in the spectrofluorimeter. Thus, the absolute fluorescence quantum yields in solid samples are only approximate. Nevertheless, from this analysis the relative fluorescence efficiency between different solid samples can be obtained. The photophysical parameters of samples with high dye concentration are corrected by reabsorption/reemission phenomena through appropriate mathematical procedures leading to $\lambda_{\mathrm{em}}, \phi$ and $\tau$ values similar to those observed in diluted dye<smiles>C=C(C)C(=O)OC</smiles><smiles>C=C(C)OCO</smiles>

(MMA)<smiles>C=CC(=O)OCOc1ccccc1</smiles><smiles>C=CN1CCCC1=O</smiles>

(PEA)
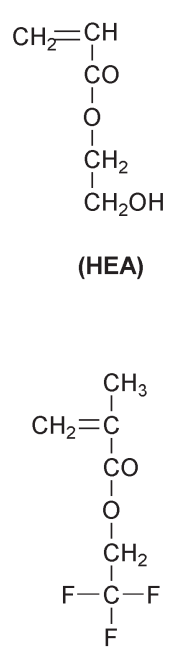

(TFMA)
Scheme 4 Molecular structures of monofunctional monomers considered in this work.<smiles>C=C(C)C(=O)OCC(=O)OCC(CC)(COC(=O)C(=C)C)COC(=O)C(=C)C</smiles>

EGDMA

TMPTMA

$$
\mathrm{H}_{2} \mathrm{C}=\mathrm{CH}-\mathrm{COO}-\left[\mathrm{CH}_{2}-\mathrm{CH}_{2}-\mathrm{O}\right]_{4} \mathrm{OC}-\mathrm{CH}=\mathrm{CH}_{2}
$$

TEGDA

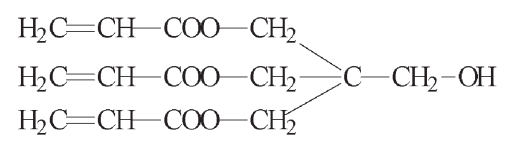

PETA

$$
\begin{aligned}
& \mathrm{H}_{2} \mathrm{C}=\mathrm{CH}-\mathrm{COO}-\mathrm{CH}_{2} \\
& \mathrm{H}_{2} \mathrm{C}=\mathrm{CH}-\mathrm{COO}-\mathrm{CH}_{2}-\mathrm{C}-\mathrm{CH}_{2}-\mathrm{OOC}-\mathrm{CH}=\mathrm{CH}_{2}
\end{aligned}
$$

Scheme 5 Molecular structures of cross-linking monomers considered in this work: methacrylic (EGDMA, TMPTMA) and acrylic (TEGDA, PETA, PETRA).

solutions. The $\phi$ values of the new dipyrromethene derivatives into polymeric matrices were evaluated relative to the dye PM567 in a solid disk of a copolymer of trifluoromethyl methacrylate $(30 \%)$ and methyl methacrylate $(70 \%)$, under similar experimental conditions. ${ }^{38}$

Radiative decay curves were recorded by the time-correlated single photon counting technique (Edinburgh Instruments model $\eta F 900)$. The emission was monitored at about $540 \mathrm{~nm}$ after excitation at 490 or $510 \mathrm{~nm}$ by means of a hydrogen flash-lamp with $1.5 \mathrm{~ns}$ FWHM pulses and $40 \mathrm{kHz}$ repetition rate. The photophysical properties in diluted dye solutions $\left(2 \times 10^{-6} \mathrm{~mol}^{-1}\right)$ were studied in $1-\mathrm{cm}$ optical pathlength quartz cuvettes. The fluorescence decay curves were analysed as a one-exponential decay $\left(\chi^{2}<1.2\right)$ and the fluorescence decay time $(\tau)$ was obtained from the slope.

The fluorescence spectra and decay curves of more concentrated solutions (up to $10^{-3} \mathrm{~mol}^{-1}$ ) were recorded in 0.1 , 0.01 and $0.001 \mathrm{~cm}$ optical path quartz cuvettes using the front-face configuration, orientating the cuvettes $35^{\circ}$ and $55^{\circ}$ with respect to the excitation and the emission-detection beams, respectively, in order to minimize the reabsorption and reemission effects. ${ }^{39}$

The estimated errors in the determination of $\phi$ and $\tau$ values are $5 \%$ and $1 \%$, respectively. The rate constants for the radiative $\left(k_{\mathrm{ff}}\right)$ and non-radiative $\left(k_{\mathrm{nr}}\right)$ deactivation processes were calculated as: $k_{\mathrm{fl}}=\phi / \tau$ and $k_{\mathrm{nr}}=(1-\phi) / \tau$. The temperature was always $20 \pm 0.2^{\circ} \mathrm{C}$. All measurements were performed in aired samples.

Details of the experimental set-up to determine the absorption cross-section and stimulated emission cross-section spectra, fluorescence quantum distributions, and degrees of fluorescence polarization as well as the saturable absorption behaviour due to picosecond laser pulse excitation and the 
photo-degradation under $\mathrm{cw}$ laser excitation and under pulsed picosecond pulse excitation can be found in ref. 40 .

\subsection{Photophysical properties of dye PM567 in liquid solution}

The photophysics of PM567 (Scheme 2) in liquid phase was characterized by using a variety of solvents which can be roughly classified as hydrogen-bond donor (HBD) (or "protic") solvents, dipolar non-HBD (or "aprotic") solvents and apolar non-HBD solvents. ${ }^{41}$

The VIS absorption band of PM567 in methanol solution is centred at $\lambda_{\max }=516 \mathrm{~nm}$ (molar absorption coefficient $=$ $\left.(7.9 \pm 0.2) \times 10^{4} \mathrm{Mol}^{-1} . \mathrm{cm}^{-1}\right)$ and its shape (Fig. 1) is very similar to that of rhodamine dyes. ${ }^{37}$ Dipyrromethene. $\mathrm{BF}_{2}$ complexes have been identified as cyclic cyanine chromophores. $^{25}$

The shape of the PM567 absorption band in methanol does not appreciably change with the dye concentration from $10^{-6}$ mol $1^{-1}$ (optical path $=1 \mathrm{~cm}$ ) to $7.7 \times 10^{-4}$ mol $1^{-1}$ (optical path $=0.01 \mathrm{~cm})$, indicating that the aggregation of dye molecules is negligible up to $\approx 10^{-3} \mathrm{~mol} 1^{-1}$. This is an important advantage with respect to rhodamine dyes, in which the aggregation of the dye is easily detected, leading to self-quenching of the monomer emission, a drastically decrease of the fluorescence quantum yield ${ }^{42}$ and an increase of the losses in the laser resonant cavity at high concentrated dye solutions. ${ }^{43}$

The fluorescence spectrum of PM567 in methanol (Fig. 1) is nearly the mirror image of the absorption band, with a Stokes shift of about $\Delta \nu \approx 560 \mathrm{~cm}^{-1}$. The shape of this spectrum is independent of the excitation wavelength, indicating that the fluorescence emission is from the lowest vibrational level of the $S_{1}$ excited state, even if the excitation is performed to the second electronic excited state $\left(\lambda_{\mathrm{ex}}<400 \mathrm{~nm}\right)$. The fluorescence quantum yield $(\phi)$ of a diluted solution of PM567 in methanol is 0.91 . The fluorescence decay curve of this sample can be analysed as a single-exponential deactivation curve with a fluorescence lifetime $(\tau)$ of $6.1 \mathrm{~ns}^{41}$

The photophysical characteristics of PM567 in liquid solution are affected by the solvent. Table 1 summarizes the absorption and fluorescence properties of this dye in several solvents. Laser data, which are also included in the table, will be analysed in section 4.2. Some interesting tendencies can be derived from the experimental data: higher $\phi$ and lower $\tau$ values are observed for very polar protic solvents, and lower $\phi$ are obtained in aromatic solvents with both apolar and protic characteristics. Probably, a $\pi$-electron interaction between the excited state of the dye and the aromatic ring of the solvent ${ }^{44}$ is the responsible for this loss in the fluorescence

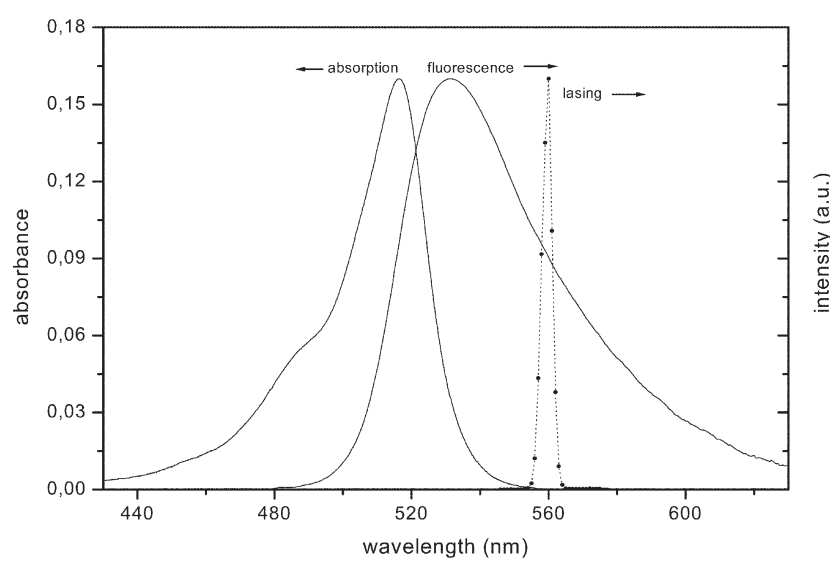

Fig. 1 UV/VIS absorption and normalized fluorescence spectra of $2 \times 10^{-6} \mathrm{~mol}^{-1}$ solution of PM567 in methanol at $20^{\circ} \mathrm{C}$. The lasing emission spectra of PM567 $1 \times 10^{-3} \mathrm{~mol}^{-1}$ in methanol is also included. quantum yield of PM567 in these solvents. Thus, aromatic solvents can be apparently catalogued as non-recommended media for laser action of PM567. Although $\phi$ and $\tau$ values do not linearly correlate with any solvent parameters, it is generally observed (Table 1) an increase in both values when changing from apolar to protic solvents (i.e from $\phi=0.97$ and $\tau=6.94 \mathrm{~ns}$ in 2,2,2-trifluoroethanol to $\phi=0.70$ and $\tau=5.60 \mathrm{~ns}$ in cyclohexane). This decrease in $\phi$ and $\tau$ values is mainly due to an increase in the non-radiative deactivation process rather than to a fall in the fluorescence probability. ${ }^{41}$ The knowledge of the non-radiative deactivation mechanism is fundamental in laser dye systems since it rules the fluorescence quantum yield, and, ultimately, the lasing efficiency of the dyes. For the PM567 system, one could think that the decrease in $k_{\mathrm{nr}}$ value in polar-protic solvents is related to a change in the distribution of the positive charge through the cyclic cyanine $\pi$-system. In this sense, all dye-solvent interactions affecting the distribution of the positive charge in the dipyrromethene $\mathrm{BF}_{2}$ complex will decrease the non-radiative deactivation. On the other hand, a H-bond interaction between the electron-lone pair of the amino groups of the dye and protic solvents would decrease the internal conversion of PM567 in protic solvent. ${ }^{41}$

Thus, for laser operations of PM567 in liquid solution the use of polar-protic media is recommended, although a slight decrease in the molar absorption coefficient in these solvents (Table 1) will imply a somewhat less efficient pumping process. Moreover, the largest Stokes shift values are observed in protic solvents, with the corresponding decrease in the loss of fluorescence intensity by reabsorption and reemission effects in concentrated dye solutions.

\subsection{Photophysical properties of dye PM567 in solid polymeric matrices}

In our previous work on rhodamines, an apparent direct relationship between photophysical and lasing properties of dyes in linear polymeric materials had been observed: the higher the lasing efficiency, the higher the fluorescence rate constant and the lower the nonradiative rate constant. ${ }^{45}$ At this time, it was worthy to know if this behaviour was also followed in dye doped crosslinked polymeric matrices. To this aim, a systematic analytical study of the photophysical properties of PM567 incorporated into solid samples of 2,2,2,-trifluoromethyl methacrylate (TFMA, Scheme 4), monomer that mimics the best liquid media for PM567 laser operation (that is, 2,2,2-trifluoroethanol, Table 1), with different amounts of the crosslinking monomer ethylene glycol dimethacrylate (EGDMA, Scheme 5) at the dye concentration needed for efficient laser operation was carried out. ${ }^{38}$ The main photophysical characteristics of these systems are reported in Table 2.

The VIS absorption band of PM567 dissolved in these matrices shifts slowly to lower energies as the molar proportion of EGDMA increases in the polymeric matrix. The shape of the absorption band is very similar to that registered in ethanol and does not appreciably change with the proportion of the crosslinker monomer. To put these facts into proper perspective, measurements were made of the photophysical properties of PM567 dissolved in copolymer P(MMA:TFMA $70: 30)$, to analyse the influence of crosslinking with respect to the lineal copolymer. The presence of EGDMA induces a blue-shift of the absorption maximum and reduces up to $50 \%$ the absorption capability of the dye with respect to the copolymer solution (Table 2). These photophysical aspects could reduce the effectiveness of the laser action of PM567 solved in crosslinked TFMA matrices with respect to that obtained in the copolymer P(MMA:TFMA 70:30), since the decrease in the molar absorption coefficient requires to use a more concentrated dye solution to reach the threshold for laser action. In addition, if the same dye-polymer interactions in the 
Table 1 Photophysical and lasing properties of PM567 in several solvents at $20^{\circ} \mathrm{C}$ : absorption, fluorescence, and lasing maximum wavelength $\left(\lambda_{\mathrm{ab}}, \lambda_{\mathrm{fl}}, \lambda_{\mathrm{la}}\right)$, molar absorption coefficient $\left(\varepsilon_{\mathrm{max}}\right)$, fluorescence quantum yield $(\phi)$, lifetime $(\tau)$ and percent lasing efficiency (Eff) (under pumping with $5.5 \mathrm{~mJ}$ pulses from a Ng:KGW laser (second harmonic)). [PM567] $=2 \times 10^{-6} \mathrm{~mol}^{-1}$ in absorption and fluorescence data and $1 \times 10^{-3}$ $\mathrm{mol}^{-1}$ in lasing data. Data taken from ref. 41

\begin{tabular}{|c|c|c|c|c|c|c|c|}
\hline Solvent $^{a}$ & $\lambda_{\mathrm{ab}} / \mathrm{nm}$ & $10^{-4} \varepsilon_{\max } / \mathrm{M}^{-1} \mathrm{~cm}^{-1}$ & $\lambda_{\mathrm{fl}} / \mathrm{nm}$ & $\phi$ & $\tau / \mathrm{ns}$ & $\lambda_{\mathrm{la}}^{b} / \mathrm{nm}$ & Eff $(\%)$ \\
\hline 1 2-Methylbutane (npa) & 520.4 & 9.6 & 534.4 & 0.87 & 6.28 & $c$ & $c$ \\
\hline $2 n$-Hexane (npa) & 521.1 & 9.2 & 533.6 & 0.83 & 5.90 & 566 & 35 \\
\hline 3 Cyclohexane (npa) & 522.5 & 9.3 & 537.2 & 0.70 & 5.60 & 571 & 28 \\
\hline 4 Isooctane (npa) & 521.2 & 9.4 & 535.2 & 0.77 & 5.84 & 568 & 30 \\
\hline 5 Benzene (npa) & 522.7 & 8.5 & 536.4 & 0.59 & 5.00 & 569 & 24 \\
\hline 6 Toluene (npp) & 522.5 & 8.6 & 536.4 & 0.50 & 5.07 & 569 & 22 \\
\hline 7 Diethyl ether (npp) & 518.8 & 8.7 & 532.8 & 0.89 & 6.21 & 565 & 48 \\
\hline 8 1,4-Dioxane (npa) & 518.6 & 8.4 & 533.2 & 0.83 & 5.48 & 566 & 34 \\
\hline 9 Tetrahydrofurane (npp) & 518.4 & 8.3 & 534.0 & 0.75 & 5.63 & $c$ & $c$ \\
\hline 10 1-Methylpyrrolidin-2-one (npp) & 518.2 & 5.8 & 533.2 & 0.57 & 5.56 & 565 & 28 \\
\hline 11 Ethyl acetate (npp) & 516.4 & 8.0 & 531.6 & 0.80 & 5.78 & 563 & 33 \\
\hline $12 N, N$-Dimethylformamide (npp) & 517.1 & 7.0 & 532.8 & 0.67 & 5.57 & $c$ & $c$ \\
\hline 13 Acetone (npp) & 516.0 & 8.0 & 532.0 & 0.85 & 5.92 & 564 & 42 \\
\hline 14 Acetonitrile (npp) & 514.8 & 7.9 & 530.4 & 0.87 & 5.96 & 562 & 46 \\
\hline 15 Ethane-1,2-diol (pp) & 518.0 & 7.5 & 536.4 & 0.71 & 5.80 & $c$ & $c$ \\
\hline 16 2,2,2-Trifluoroethanol (pp) & 515.7 & 7.1 & 535.2 & 0.97 & 6.94 & 563 & 56 \\
\hline 17 2-Phenoxyethanol (pp) & 522.3 & 7.7 & 536.8 & 0.61 & 5.46 & 570 & 34 \\
\hline 18 1-Decanol (pp) & 521.8 & 8.4 & 534.8 & 0.77 & 5.71 & $c$ & $c$ \\
\hline 19 1-Octanol (pp) & 521.0 & 8.1 & 534.4 & 0.71 & 5.90 & 567 & 27 \\
\hline 20 1-Hexanol (pp) & 520.4 & 8.5 & 534.8 & 0.76 & 5.78 & 567 & 29 \\
\hline 21 1-Butanol (pp) & 519.4 & 8.2 & 534.4 & 0.76 & 5.98 & $c$ & $c$ \\
\hline 22 2-Propanol (pp) & 518.8 & 8.2 & 534.0 & 0.83 & 6.04 & $c$ & $c$ \\
\hline 23 1-Propanol (pp) & 518.9 & 8.0 & 534.0 & 0.78 & 6.01 & 567 & 33 \\
\hline 24 Ethanol (pp) & 517.7 & 8.1 & 532.4 & 0.84 & 6.09 & 565 & 37 \\
\hline 25 Methanol (pp) & 516.2 & 7.9 & 531.6 & 0.91 & 6.10 & 563 & 52 \\
\hline
\end{tabular}

${ }^{a}$ In parenthesis after the name of each solvent; pp: protic polar (hydrogen-bond donor (HBD) or "protic"); npp: non-protic polar (dipolar non-HBD or "aprotic"); npa: non-protic apolar (apolar non-HBD). ${ }^{b}$ All the wavelengths are increased in 2 nm with respect to those quoted in ref. 41 because of recalibration of the detector. ${ }^{c}$ Not measured.

ground and the excited state are considered, a decrease in the integrated molar absorption coefficient value would imply a decrease in the fluorescence rate constant, according to the Strickler and Berg equation. ${ }^{46}$

The photophysical properties of PM567 dissolved in TFMA are critically dependent on the different amounts of the crosslinking monomer EGDMA. The presence of EGDMA at low molar proportion initially induces a reduction in both the fluorescence quantum yields and lifetime, reaching a minimum for the (TFMA:EGDMA 98:2) sample. An augmentation of the proportion of EGDMA improves the photophysics of these materials, with the highest fluorescence quantum yield being reached with the sample with a molar ratio of $20 \%$. The experimental results of Table 2 suggest that PM567 incorporated into TFMA:EGDMA $20 \%$ could be a very attractive material as active media in laser technological applications since the fluorescence rate constant of the dye increases a $20 \%$ whereas the non-radiative rate constant decreases by a factor 10 with respect to the values reached for these parameters in the lineal copolymer MMA:TFMA 70:30. On the other hand, factors such as the smaller absorption coefficient (diminution of the pumping efficiency) and a smaller Stokes shift (augmentation of the reabsorption/reemission probability in high concentrated dye samples) would be expected to somehow reduce the lasing efficiency of PM567 in this crosslinked matrix.

\subsection{Photophysical properties of dyes PnAC in liquid phase}

The photophysical properties of the novel PnAc $(n=$ $1,3,5,10,15$; Scheme 2) dyes were obtained by recording their absorption and fluorescence spectra and the fluorescence decay curves. ${ }^{47}$ Fig. 2 shows representative spectra for the dyes with the shortest (P1Ac) and the longest (P15Ac) 8-polymethylene chain. Generally speaking, the shape and intensity of the absorption and fluorescence bands are similar to those of the

Table 2 Photophysical properties of PM567 dissolved in both the linear copolymer composed of MMA:TFMA 7:3 and in the crosslinked copolymers of TFMA with different amounts of EGDMA: absorption and fluorescence maximum wavelengths $\left(\lambda_{\mathrm{ab}}\right.$ and $\left.\lambda_{\mathrm{em}}\right)$; molar absorption coefficient at the absorption maximum $(\varepsilon)$, fluorescence quantum yield $(\phi)$; lifetime $(\tau)$. Dye concentration: $1.5 \times 10^{-3}$ mol $1^{-1}$. Optical pathway: $0.2 \mathrm{~mm}$. Data taken from ref. 38

\begin{tabular}{|c|c|c|c|c|c|c|c|c|}
\hline Material & $\lambda_{\mathrm{ab}} / \mathrm{nm}$ & $10^{-4} \varepsilon / \mathrm{M}^{-1} \mathrm{~cm}^{-1}$ & $\lambda_{\mathrm{em}} / \mathrm{nm}$ & $\phi$ & $\tau / \mathrm{ns}$ & $\lambda_{\mathrm{em}}{ }^{a} / \mathrm{nm}$ & $\phi^{a}$ & $\tau^{a} / \mathrm{ns}$ \\
\hline MMA:TFMA 70:30 & 518.0 & 6.97 & 543.0 & 0.644 & 9.81 & 538.0 & 0.805 & 7.85 \\
\hline TFMA:EGDMA 99:1 & 517.1 & 4.75 & 539.8 & 0.643 & 9.67 & 534.8 & 0.804 & 7.74 \\
\hline TFMA:EGDMA 98:2 & 517.3 & 4.13 & 538.6 & 0.602 & 9.44 & 533.6 & 0.753 & 7.55 \\
\hline TFMA:EGDMA 95:5 & 517.3 & 3.41 & 538.6 & 0.724 & 9.60 & 533.6 & 0.905 & 7.68 \\
\hline TFMA:EGDMA 90:10 & 517.4 & 4.03 & 538.6 & 0.722 & 9.59 & 533.6 & 0.903 & 7.67 \\
\hline TFMA:EGDMA 80:20 & 517.7 & 4.67 & 538.6 & 0.783 & 9.71 & 533.6 & 0.979 & 7.77 \\
\hline
\end{tabular}




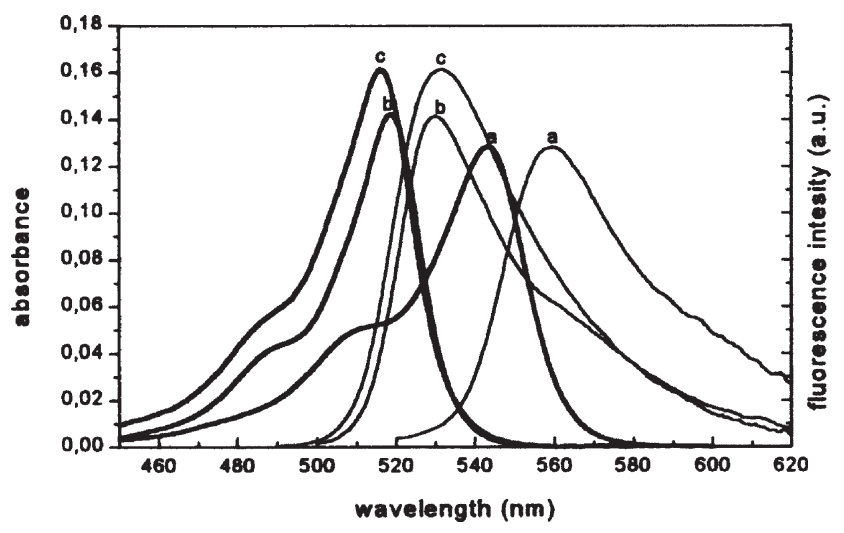

Fig. 2 Absorption (bold curves) and corrected and normalized fluorescence (fine curves) spectra of diluted solutions $\left(2 \times 10^{-6} \mathrm{M}\right)$ of P1Ac (a), P15Ac (b) and PM567(c) in methanol (1 cm optical pathway cuvette).

commercial dye PM567, ${ }^{41}$ indicating that the presence of the 8 -acetoxymethyl group does not extensively modify the electronic $\pi$-system of the chromophore. Table 3 summarizes the photophysical data of diluted solutions of all the new dyes in several solvents.

The dye P1Ac presents the most drastic changes in the photophysical properties with respect to those of the commercial dye PM567: both absorption and fluorescence bands are bathochromically shifted around $1000 \mathrm{~cm}^{-1}$, the fluorescence quantum yield is lower and the lifetime is higher indicating a diminution in the radiative deactivation rate constant and an augmentation in the non-radiative deactivation process. The diminution in the spectroscopic transition probability between the $\mathrm{S}_{0}-\mathrm{S}_{1}$ electronic states is also deduced from the absorption data, since the oscillator strength (measure for integrated intensity of electronic transitions) of P1Ac is smaller than that of PM567 in all the studied solvents. The photophysics of the other $\operatorname{PnAc}(n=3,5,10$ and 15$)$ dyes progressively mimics that of PM567 dye as the length of the 8-polymethylene chain increases. Exception is the position of the fluorescence band which exhibits, for some of the dyes in certain solvents, a small hypsochromic shift, up to $240 \mathrm{~cm}^{-1}$, with respect to the position of the emission band of PM567 (Table 3).

These experimental results suggest that the effect of the $\omega$-acetoxy group on the photophysics of the PM567 chromophore is not due to a direct intramolecular through-space interaction between the ester group and the $\pi$-electrons of the aromatic rings favoured by the length and the flexibility of the 8-polymethylene chain, but rather to the electron withdrawing effect of the $\omega$-acetoxy group through the linking chain. These arguments are supported by theoretical calculations. ${ }^{47}$

The effects of the solvent on the photophysics of these dyes are nearly independent of the 8-chain length and they are similar to those observed for the PM567 dye (Table 1). From the data listed in Table 3 , it can be concluded that the incorporation to the chain of a polymerizable ester end group can provide the way to covalently link the PM567 chromophore to a polymer chain without drastic modifications in the photophysics of the dye. These are essential aspects in the development of active media for solid-state tunable dye laser. ${ }^{7,17}$

The photophysical characteristics of $\mathrm{P} n \mathrm{Ac}$ dyes as a function of the dye concentration were also studied. The shape of the absorption spectrum of $\mathrm{P} n \mathrm{Ac}$ derivatives is independent of the dye concentration up to a maximum concentration of

Table 3 Photophysical properties of diluted solutions $\left(2 \times 10^{-6} \mathrm{~mol} \mathrm{l}^{-1}\right.$ in $1 \mathrm{~cm}$ pathway cuvettes $)$ of the dyes PnAc in different solvents: absorption and fluorescence wavelengths $\left(\lambda_{\mathrm{ab}}\right.$ and $\left.\lambda_{\mathrm{fl}}\right)$, oscillator strength $(f)$, fluorescence quantum yield $(\phi)$, and lifetime $(\tau)$. Data taken from ref. 47

\begin{tabular}{|c|c|c|c|c|c|c|}
\hline Dye & Solvent & $\lambda_{\mathrm{ab}}( \pm 0.1 \mathrm{~nm})$ & $\lambda_{\mathrm{fl}}( \pm 0.1 \mathrm{~nm})$ & $f$ & $\phi( \pm 0.05)$ & $\tau( \pm 0.05 \mathrm{~ns})$ \\
\hline \multirow[t]{6}{*}{ P1Ac } & $\mathrm{F}_{3}$-ethanol ${ }^{a}$ & 545.6 & 562.5 & 0.371 & 0.74 & 7.85 \\
\hline & Methanol & 543.2 & 559.0 & 0.409 & 0.68 & 6.65 \\
\hline & Ethanol & 544.4 & 560.0 & 0.374 & 0.66 & 6.56 \\
\hline & Acetone & 542.4 & 557.5 & 0.385 & 0.69 & 6.86 \\
\hline & Ethyl acetate & 542.9 & 558.0 & 0.372 & 0.68 & 6.64 \\
\hline & Cyclohexane & 547.6 & 561.0 & 0.424 & 0.56 & 6.27 \\
\hline \multirow[t]{6}{*}{$\mathrm{P} 3 \mathrm{Ac}$} & $\mathrm{F}_{3}$-ethanol ${ }^{a}$ & 519.9 & 531.5 & 0.396 & 0.91 & 7.05 \\
\hline & Methanol & 520.0 & 532.0 & 0.414 & 0.81 & 6.06 \\
\hline & Ethanol & 521.2 & 533.0 & 0.418 & 0.77 & 5.93 \\
\hline & Acetone & 519.5 & 531.0 & 0.397 & 0.80 & 5.98 \\
\hline & Ethyl acetate & 520.1 & 531.0 & 0.420 & 0.75 & 5.88 \\
\hline & Cyclohexane & 524.9 & 533.5 & 0.433 & 0.58 & 5.54 \\
\hline \multirow[t]{6}{*}{$\mathrm{P} 5 \mathrm{Ac}$} & $\mathrm{F}_{3}$-ethanol ${ }^{a}$ & 518.0 & 529.0 & 0.410 & 0.95 & 7.21 \\
\hline & Methanol & 519.1 & 530.5 & 0.460 & 0.85 & 6.19 \\
\hline & Ethanol & 520.4 & 531.5 & 0.455 & 0.78 & 5.98 \\
\hline & Acetone & 518.6 & 530.5 & 0.470 & 0.76 & 6.02 \\
\hline & Ethyl acetate & 519.3 & 530.5 & 0.464 & 0.76 & 5.95 \\
\hline & Cyclohexane & 524.0 & 533.5 & 0.452 & 0.57 & 5.71 \\
\hline \multirow[t]{6}{*}{$\mathrm{P} 10 \mathrm{Ac}$} & $\mathrm{F}_{3}$-ethanol ${ }^{a}$ & 517.1 & 528.5 & 0.412 & 0.97 & 7.11 \\
\hline & Methanol & 518.6 & 530.5 & 0.418 & 0.86 & 6.39 \\
\hline & Ethanol & 520.0 & 531.0 & 0.423 & 0.80 & 5.99 \\
\hline & Acetone & 518.2 & 529.5 & 0.422 & 0.80 & 5.98 \\
\hline & Ethyl acetate & 519.0 & 530.0 & 0.420 & 0.78 & 5.92 \\
\hline & Cyclohexane & 523.1 & 533.0 & 0.437 & 0.61 & 5.68 \\
\hline \multirow[t]{6}{*}{ P15Ac } & $\mathrm{F}_{3}$-ethanol ${ }^{a}$ & 517.0 & 528.5 & 0.438 & 0.95 & 7.07 \\
\hline & Methanol & 518.7 & 530.0 & 0.455 & 0.83 & 6.29 \\
\hline & Ethanol & 519.9 & 531.0 & 0.437 & 0.79 & 6.08 \\
\hline & Acetone & 518.1 & 529.5 & 0.485 & 0.76 & 6.03 \\
\hline & Ethyl acetate & 518.9 & 529.5 & 0.475 & 0.75 & 5.91 \\
\hline & Cyclohexane & 523.2 & 532.0 & 0.435 & 0.63 & 5.72 \\
\hline
\end{tabular}




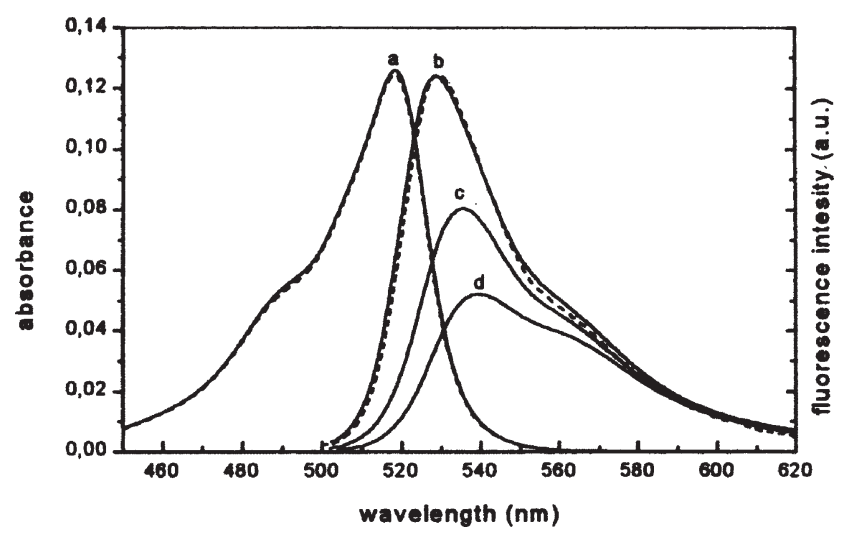

Fig. 3 Absorption (a) and corrected fluorescence (b) spectra of diluted $\left(2 \times 10^{-6} \mathrm{~mol} \mathrm{l}^{-1}\right.$ in $1 \mathrm{~cm}$ cuvette, dashed curves $)$ and concentrated $\left(2 \times 10^{-3} \mathrm{~mol} \mathrm{l}^{-1}\right.$ in $0.001 \mathrm{~cm}$ cuvette, solid curves) solutions of P5Ac in 2,2,2-trifluoroethanol. The fluorescence spectrum of the concentrated solution $\left(2 \times 10^{-3} \mathrm{~mol} \mathrm{l}^{-1}\right)$ in $0.01 \mathrm{~cm}(\mathrm{c})$ and $0.1 \mathrm{~cm}(\mathrm{~d})$ cuvettes are also included.

$2 \times 10^{-3} \mathrm{~mol} \mathrm{l}^{-1}$ (Fig. 3), suggesting the non-aggregation of the chromophores in the concentration range studied.

The fluorescence lifetimes of $\mathrm{P} n \mathrm{Ac}$ dyes in a highly concentrated $\left(10^{-3} \mathrm{~mol}^{-1}\right)$ dye solution (Table 4) are similar to those in a diluted solution (Table 3). This means that any additional (bimolecular) deactivation process from the $\mathrm{S}_{1}$ excited state does not significantly take place in concentrated dye solutions. However, moderate changes in the fluorescence quantum yield values obtained in concentrated solutions (Table 4) with respect to those in more diluted solutions (Table 3) are registered. As observed in diluted solutions, the P1Ac dye has the lowest fluorescence quantum yield, and for a common dye the lowest $\phi$ value is reached in the apolar cyclohexane solvent.

The evolution of the $\phi$ value of the dye P15Ac with the solvent in the concentrated solutions is similar to that observed in the more diluted solutions. However, the $\phi$ values of concentrated $\mathrm{P} n \mathrm{Ac}$ (with $n=1,3,5$ and 10) solutions become independent of the solvent in polar-aprotic and protic media, suggesting a poor solvent dependence of the laser efficiency in highly concentrated P $n$ Ac solutions. Different arguments were evaluated in order to explain the nearly independence of the $\phi$ value of P1Ac, P3Ac, P5Ac and P10Ac with the solvent in polar media, ${ }^{47}$ but none of them was totally satisfactory, revealing the difficulty to understand this phenomenon and at the present time we cannot offer any non-speculative explanation for this effect. Experimental and theoretical studies are currently in progress to gain more insight into the influence of the solvent on the photophysical behaviour of these dipyrromethene $\mathrm{BF}_{2}$ dyes at high concentrations.

\subsection{Photophysical properties of dyes PnAC and PnMA in solid state}

The photophysical properties of $\mathrm{P} n \mathrm{Ac}$ model dyes dissolved in poly(methyl methacrylate) (PMMA, Scheme 4) and of the $\mathrm{P} n \mathrm{MA}$ dye monomers copolymerized with methyl methacrylate (Scheme 3) were investigated and compared with their photophysical behaviour in liquid solutions. ${ }^{34,48}$

Table 5 summarizes the photophysical parameters of both $\mathrm{P} n \mathrm{Ac} / \mathrm{PMMA}$ and $\mathrm{COP}(\mathrm{P} n \mathrm{MA} / \mathrm{MMA})$ materials. The absorption and fluorescence spectra and quantum yield of these materials indicate that the terminal acetoxy group affects the photophysical properties of the parent chromophore when there is only one linking methylene group, but not when there are three or more methylene groups. The covalent linkage of the chromophore through a polymethyleneacetoxy group to a polymeric chain does not significantly alter their photophysics. On the other hand, the shape of the absorption and fluorescence bands of the polymeric samples is broader than those of the same $\mathrm{P} n \mathrm{Ac}$ analogs in liquid solution, probably due to a reduction in the vibronic structure resolution of the electronic bands of the solid samples. ${ }^{47,48}$

One of the most important photophysical aspects to emphasize for $\mathrm{P} n \mathrm{Ac}$ and $\mathrm{P} n \mathrm{MA}$ analogs in solid polymeric and copolymeric matrices, respectively, is that, except for P1Ac and P1MA, their fluorescence intensity is higher than that of the parent dye PM567 in solid PMMA. The presence of three or higher members of methylene groups at the 8-position increases the fluorescence quantum yield of the samples.

The photophysical properties of the new dyes in solid polymers (Table 5) remind those observed in polar liquid solvents, ${ }^{47}$ indicating that the surrounding of dyes in the polymer matrix corresponds to a polar environment, as expected, and that the rigidity of the solid environment does not significantly alter the photophysics of the common PM567 chromophore. The Stokes shifts in polymeric matrices are lower than those obtained in solutions, ${ }^{47}$ which can be assigned to the more rigid environment present in polymeric matrices, that inhibits the orientation of the surrounding molecules. ${ }^{49}$ The decay component is characterized by a long lifetime $(\approx 10 \mathrm{~ns})$, which represents the experimental fluorescence lifetime. This value is higher that those observed in diluted samples, ${ }^{40,47}$ which can be attributed to reabsorption and reemission phenomena occurring at high dye concentration. ${ }^{48}$

To asses the effect of dye concentration on the photophysical properties, more diluted solid samples containing P1Ac and P15Ac analogs and P1MA and P15MA monomers were prepared and their photophysics studied (Table 6). ${ }^{48}$ The decrease in the dye concentration does not lead to any changes in the absorption band, but it modifies some fluorescence characteristics: the emission band is hypsochromically shifted, the fluorescence quantum yield increases and the radiative lifetime decreases. These changes in the fluorescence parameters are

Table 4 Fluorescence quantum yield $(\phi)$ and lifetime $(\tau)$ of concentrated PnAc solutions $\left(10^{-3}\right.$ mol $\left.1^{-1}\right)$ in $0.001 \mathrm{~cm}$ pathways cells ${ }^{a}$. Data taken from ref. 47

\begin{tabular}{|c|c|c|c|c|c|c|c|c|c|c|c|c|}
\hline \multirow[b]{2}{*}{ Solvent } & \multicolumn{6}{|c|}{$\phi( \pm 0.10)$} & \multicolumn{6}{|c|}{$\tau( \pm 0.05 \mathrm{~ns})$} \\
\hline & $\mathrm{P} 1 \mathrm{Ac}{ }^{b}$ & $\mathrm{P} 3 \mathrm{Ac}$ & $\mathrm{P} 5 \mathrm{Ac}$ & P10Ac & P15Ac & PM567 & $\mathrm{P} 1 \mathrm{Ac}{ }^{b}$ & P3Ac & P5Ac & P10Ac & P15Ac & PM567 \\
\hline $\mathrm{F}_{3}$-ethanol ${ }^{c}$ & 0.64 & 0.81 & 0.83 & 0.82 & 0.85 & 0.88 & 7.70 & 7.05 & 7.29 & 7.32 & 7.08 & 6.80 \\
\hline Methanol & 0.61 & 0.77 & 0.90 & 0.88 & 0.84 & 0.91 & 6.44 & 6.10 & 6.26 & 6.33 & 6.31 & 5.78 \\
\hline Ethanol & 0.60 & 0.78 & 0.84 & 0.80 & 0.70 & 0.83 & 6.58 & 6.09 & 6.27 & 6.35 & 6.11 & 5.88 \\
\hline Acetone & 0.54 & 0.89 & 0.87 & 0.89 & 0.75 & 0.87 & 7.04 & 6.14 & 6.18 & 6.39 & 6.17 & 6.01 \\
\hline Ethyl acetate & 0.60 & 0.85 & 0.90 & 0.92 & 0.75 & 0.83 & 6.90 & 5.96 & 6.21 & 6.11 & 6.12 & 5.99 \\
\hline Cyclohexane & 0.58 & 0.63 & 0.55 & 0.58 & 0.54 & 0.69 & 6.41 & 5.61 & 5.76 & 5.94 & 5.82 & 5.64 \\
\hline
\end{tabular}

${ }^{a}$ Other photophysical parameters such as $\lambda_{\mathrm{ab}}, \lambda_{\mathrm{fl}}$, and $\mathrm{f}$ are not included in this table because they have the same values than those in diluted solutions (Table 3). Values of PM567 are included for comparison. ${ }^{b}$ Dye concentration: $0.45 \times 10^{-3}$ mol $1^{-1}$ in a 0.01 -cm cuvette. ${ }^{c} 2,2,2$-trifluoroethanol. 
Table 5 Photophysical properties of PnAc/PMMA and COP(P $n$ MA/MMA) samples (dye concentration: $1.5 \times 10^{-3}$ mol $\left.1^{-1}\right)$ : absorption and fluorescence wavelengths $\left(\lambda_{\mathrm{ab}}\right.$ and $\left.\lambda_{\mathrm{fl}}\right)$, oscillator strength $(f)$, fluorescence quantum yield $(\phi)$, and lifetime $(\tau)$. $\lambda_{\mathrm{fl}}^{\mathrm{c}}, \phi^{\mathrm{c}}$, and $\tau^{\mathrm{c}}$ are values corrected from reabsorption and reemission effects. Data for PM567 are also included for comparison. Data taken from ref. 48

\begin{tabular}{|c|c|c|c|c|c|c|c|c|}
\hline System & $\lambda_{\mathrm{ab}}( \pm 0.1 \mathrm{~nm})$ & $f$ & $\lambda_{\mathrm{fl}}( \pm 0.5 \mathrm{~nm})$ & $\lambda_{\mathrm{fl}}^{\mathrm{c}}$ & $\phi( \pm 0.05)$ & $\phi^{\mathrm{c}}$ & $\tau( \pm 0.05 \mathrm{~ns})$ & $\tau^{\mathrm{c}}$ \\
\hline PM567/PMMA & 518.5 & 0.475 & 543.2 & 531.0 & 0.53 & 0.63 & 10.39 & 8.97 \\
\hline P1Ac/PMMA & 544.5 & 0.344 & 565.0 & 556.5 & 0.48 & 0.61 & 9.95 & 8.21 \\
\hline COP(P1MA/MMA) & 546.6 & 0.378 & 566.5 & 557.0 & 0.47 & 0.62 & 10.00 & 7.96 \\
\hline P3Ac/PMMA & 521.7 & 0.520 & 545.0 & 531.5 & 0.78 & 0.89 & 10.25 & 8.97 \\
\hline COP(P3MA/MMA) & 522.4 & 0.559 & 543.5 & 532.0 & 0.71 & 0.85 & 10.51 & 8.75 \\
\hline P5Ac/PMMA & 520.8 & 0.549 & 541.5 & 530.0 & 0.70 & 0.84 & 10.65 & 9.00 \\
\hline COP(P5MA/MMA) & 521.1 & 0.533 & 542.0 & 530.0 & 0.72 & 0.85 & 10.84 & 9.10 \\
\hline P10Ac/PMMA & 520.6 & 0.538 & 541.0 & 529.5 & 0.71 & 0.84 & 10.65 & 8.93 \\
\hline COP(P10MA/MMA) & 520.7 & 0.493 & 541.0 & 529.5 & 0.69 & 0.83 & 10.58 & 8.72 \\
\hline P15Ac/PMMA & 520.6 & 0.346 & 538.5 & 529.0 & 0.62 & 0.78 & 9.97 & 7.98 \\
\hline COP(P15MA/MMA) & 520.7 & 0.283 & 538.5 & 529.5 & 0.68 & 0.81 & 9.71 & 8.23 \\
\hline
\end{tabular}

typical for reabsorption/reemission effects ${ }^{39}$ since the corrected $\lambda_{\mathrm{fl}}^{\mathrm{c}}, \phi^{\mathrm{c}}$ and $\tau^{\mathrm{c}}$ values become independent of the dye concentration.

Pyrromethene dyes P5Ac and P10Ac incorporated into solid matrices of different acrylic copolymers were subjected to a more detailed photophysical study, where parameters such as absorption cross-section and stimulated emission cross-section spectra, fluorescence quantum distributions, and degrees of fluorescence polarization were determined. ${ }^{40}$ Picosecond saturable absorption studies revealed negligible excited state absorption at the second harmonic frequency of $\mathrm{Nd}$-doped lasers. The practical absence of excited-state absorption avoids higher excited-state photodegradation, which is an important photo-degradation way for Rhodamine $6 \mathrm{G} .{ }^{50}$ Solid-state $\mathrm{cw}$ laser photo-degradation studies revealed a reasonably high $\mathrm{S}_{1}$-state photostability.

The photophysical parameters so determined for the newly synthesized dyes compare well with the commercial dye PM567, making the investigated pyrromethene dyes in acrylic copolymer materials good candidates as solid-state dye laser materials.

\subsection{Photostability under UV and visible irradiation}

The photostability of the model dyes PnAc and PM567 in both ethanol and PMMA solid solution as well as that of the solid copolymers COP(P $n$ MA-MMA) was evaluated by their comparative irradiation with UV light with a standard homemade UV-accelerometer device. ${ }^{34}$ The photodegradation of the samples was monitored by recording the UV/Vis absorption spectrum versus air (solid samples) or ethanol (liquid solutions) at different times, using the relative decrease of the absorbance at the visible maximum of each dye as a measure of its photodegradation rate.

Comparative photodegradation curves for the different materials are presented in Fig. 4, where the relative decrease of absorbance at the visible maximum of each dye, with regard to the initial absorbance, $A / A_{0}$, is plotted as a function of the irradiation time $t$. The decay curves can be represented by the empirical decay law $\ln \left(A / A_{0}\right)=-k_{\mathrm{d}} t$, where $k_{\mathrm{d}}$ can be considered as the experimental rate constant for the photodegradation of the chromophore. Values of $k_{\mathrm{d}}$ were obtained for each dye from linear regression analysis of points in the time range were the former expression was fulfilled (Table 7).

From the results shown in Table 7, it can be concluded that all $\mathrm{P} n \mathrm{Ac}$ dyes with three or more methylene groups in position 8 show similar photostability in ethanol solution, and that this photostability is always higher than that of the dyes P1Ac and PM567 in the same solvent. In addition, in all cases the borondipyrromethene chromophore bound to a linear polymeric chain of PMMA is more photostable than the corresponding model dye or the parent dye PM567 in PMMA solution, confirming the stabilizing effect of copolymerization. On the other hand, all the copolymers $\mathrm{COP}(\mathrm{P} n \mathrm{MA}-\mathrm{MMA})$ show not very different $k_{\mathrm{d}}$ values, indicating that the length of the methylene chain through which the chromophore is bounded to the MMA polymer has low or null influence on the photostability, under the conditions herein employed.

The photostability of the new PnAc dyes was also characterized under experimental conditions identical to that selected for laser irradiation of solid samples. ${ }^{47}$ Methyl isobutyrate was selected as the solvent because it mimics adequately the MMA monomer present in most of the solid polymeric formulations tested in our previous work. Because $8 \mu \mathrm{L}$ was estimated to be the irradiated volume in the solid samples under our experimental conditions, capillary tubes into which the liquid solutions were incorporated were used to best reproduce the irradiation conditions in the solid samples, thus maintaining the same laser pump conditions in both cases. Monitoring of sample photolysis was carried out by recording the laser induced fluorescence emission, excited transversely to the capillary tube with the same pump pulses from the Nd:YAG laser used for producing dye laser emission.

Although the optical quality of the capillary prevents laser emission from the dipyrromethene $\mathrm{BF}_{2}$ dyes tested, information about the amount of bleached molecules can be obtained by monitoring the decrease in laser induced fluorescence intensity as a function of the number of pump laser pulses. The results obtained from the dyes P1Ac and P3Ac are plotted in Fig. 5. To put these results in proper perspective,

Table 6 Photophysical properties for polymer matrices with reduced dye concentrations $(C)$ of samples with analogs P1 and P15: absorption and fluorescence wavelengths $\left(\lambda_{\mathrm{ab}}\right.$ and $\left.\lambda_{\mathrm{fl}}\right)$, oscillator strength $(f)$, fluorescence quantum yield $(\phi)$, and lifetime $(\tau)$. $\lambda_{\mathrm{fl}}^{\mathrm{c}}, \phi^{\mathrm{c}}$, and $\tau^{\mathrm{c}}$ are values corrected from reabsorption and reemission effects. Data taken from ref. 48

\begin{tabular}{|c|c|c|c|c|c|c|c|c|c|}
\hline System & $C\left(10^{-3} \mathrm{~mol} \mathrm{l}^{-1}\right)$ & $\lambda_{\mathrm{ab}}( \pm 0.1 \mathrm{~nm})$ & $f$ & $\lambda_{\mathrm{fl}}( \pm 0.5 \mathrm{~nm})$ & $\lambda_{\mathrm{fl}}^{\mathrm{c}}$ & $\phi( \pm 0.05)$ & $\phi^{\mathrm{c}}$ & $\tau( \pm 0.05 \mathrm{~ns})$ & $\tau^{\mathrm{c}}$ \\
\hline P1Ac/PMMA & 0.45 & 544.9 & 0.442 & 563.0 & 557.0 & 0.55 & 0.63 & 9.41 & 8.55 \\
\hline COP(P1MA/MMA) & 0.45 & 547.0 & 0.329 & 561.2 & 557.0 & 0.58 & 0.64 & 9.34 & 8.69 \\
\hline P15Ac/PMMA & 0.60 & 520.5 & 0.570 & 537.0 & 528.5 & 0.67 & 0.79 & 9.97 & 8.44 \\
\hline COP(P15MA/MMA) & 0.60 & 520.7 & 0.601 & 537.5 & 529.0 & 0.67 & 0.80 & 9.84 & 8.32 \\
\hline
\end{tabular}




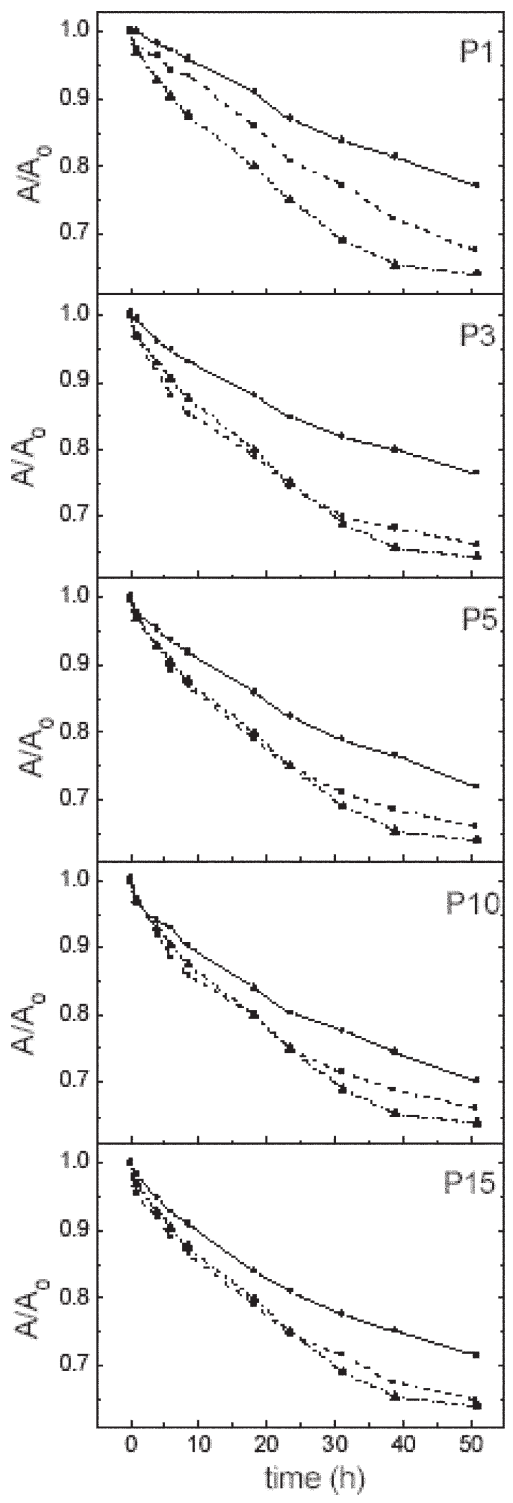

Fig. 4 Comparative photodegradation under UV irradiation of dipyrromethene $\mathrm{BF}_{2}$ dyes with 1 (P1), 3 (P3), 5 (P5), 10 (P10) and 15 (P15) methylene groups in the chain in position 8 , as solid solutions in PMMA (PnAc/PMMA, dashed lines) or as copolymers with MMA (COP(P $n$ MA-MMA), solid lines). The comparative photodegradation of the dye PM567 in solution in PMMA (dashed-dotted lines) is also included in all the plots. Irradiation at $310 \mathrm{~nm}, 1 \mathrm{~mm}$ thick disks with absorbance at the visible maximum of $c a .2$.

Table 7 Photodegradation rate constants, $k_{\mathrm{d}}$, of PM567 and the model dyes $\mathrm{P} n \mathrm{Ac}$ in ethanol solution, in solid solutions of PMMA and in the solid copolymers (COP) of methacrylate tethered monomers $\mathrm{P} n$ MA with methyl methacrylate (MMA). Irradiation at $310 \mathrm{~nm}, 1 \mathrm{~mm}$ thick disks (solids) or $1 \mathrm{~cm}$ pathlength quartz cuvettes; absorbances at the visible maximum $c a$. 2. Data taken from ref. 34

\begin{tabular}{ccll}
\hline \multicolumn{5}{c}{$k_{\mathrm{d}} / 10^{-6} \mathrm{~s}^{-1}$} \\
\cline { 2 - 4 }$n$ & $\begin{array}{l}\text { Liquid solutions } \\
\text { PnAc/EtOH }\end{array}$ & $\begin{array}{l}\text { Solid copolymers } \\
\text { COP(PnMA-MMA) }\end{array}$ & $\begin{array}{l}\text { Solid solutions } \\
\text { PnAc/PMMA }\end{array}$ \\
\hline 1 & $>40^{a}$ & $1.7 \pm 0.1$ & $2.6 \pm 0.2$ \\
3 & $3.8 \pm 0.4$ & $1.7 \pm 0.1$ & $3.8 \pm 0.5$ \\
5 & $3.8 \pm 0.4$ & $2.3 \pm 0.1$ & $2.7 \pm 0.3$ \\
10 & $3.3 \pm 0.2$ & $2.4 \pm 0.2$ & $3.0 \pm 0.2$ \\
15 & $3.2 \pm 0.1$ & $2.5 \pm 0.1$ & $2.6 \pm 0.1$ \\
PM567 & $17.2 \pm 0.6$ & & $2.9 \pm 0.1$ \\
$a$ & \\
\multicolumn{3}{l}{ The fast photodegradation avoids $k_{\mathrm{d}}$ calculation. } \\
\hline
\end{tabular}

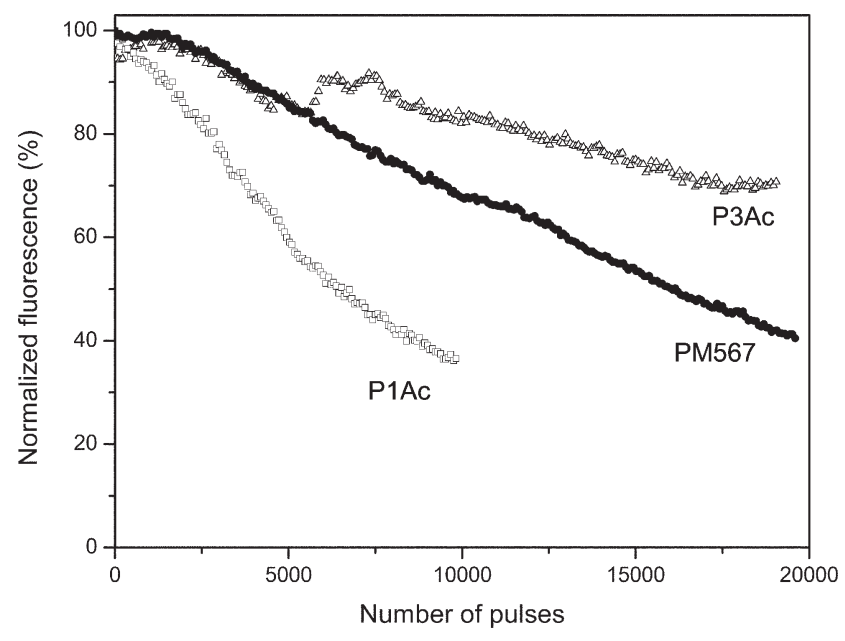

Fig. 5 Normalized laser induced fluorescence emission as a function of the number of pump pulses ( $5 \mathrm{~mJ}, 7 \mathrm{~ns}$ FWHM) at $10 \mathrm{~Hz}$ repetition rate for the dyes P1Ac, P3Ac and PM567 in methyl isobutyrate

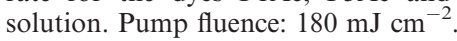

measurements were made of the degradation rate of the fluorescence emission from PM567 under the same experimental conditions. The results indicate that P3Ac is more photostable than the reference dye PM567, while P1Ac is more easily photodegraded, in good agreement with results reached previously. The presence of an $\omega$-acetoxypolymethylene chain in position 8 of the dipyrromethene $\mathrm{BF}_{2}$ core photostabilizes the chromophore in ethanol solution, with regard to the parent dye PM567, if the chain has at least three methylenes. The analysis of the laser induced fluorescence spectra before and after irradiation reveals that the photodegradation is an irreversible process leading to the generation of products in low concentration or without significant absorption at the laser irradiation wavelength, since no new features appear in the spectrum registered after irradiation. ${ }^{47}$

To gain a better insight into the degradation mechanism of these new dyes, the photostability of some materials under intense picosecond pulse excitation $\left(\lambda_{L}=527 \mathrm{~nm}\right)$ was studied by multiple intense pulse excitation of the same spot of the sample and subsequent small-signal transmission measurement after strong pump pulse attenuation. ${ }^{40}$ It was found no significant $\mathrm{S}_{n}$-state photodegradation because of negligible excited state absorption. The steady-state photodegradation due to $\mathrm{S}_{1}$-state excitation is studied by $\mathrm{cw}$ Ar-ion laser excitation involving $514.5 \mathrm{~nm}$ and $488 \mathrm{~nm}$ radiation, whereby the rise of transmission with exposure time is observed. Photodegradation quantum yields of P5Ac and P10Ac as solid solutions in some acrylate and methacrylate polymers, were estimated to be in the range $(2-6) \times 10^{-6}$. $^{4}$ These favourable photostability results are a further indication of the great possibilities of the new pyrromethene dyes as lasing active media in solid-state dye lasers.

\section{Laser operation}

\subsection{Experimental set-up for laser studies}

Laser emission was obtained from air-equilibrated dye solutions contained in a $1 \mathrm{~cm}$ optical path silica cell or from airequilibrated polymer rods, $10 \mathrm{~mm}$ in diameter and $10 \mathrm{~mm}$ length, with a cut parallel to the axis of the cylinder defining a lateral flat surface of $4 \times 10 \mathrm{~mm}$. This surface as well as the ends of the rods were hand-polished until reasonable flat surfaces were obtained. No attempt to produce laser grade surfaces was made.

A schematic diagram of the laser system in shown in Fig. 6. The oscillator cavity consisted of a $90 \%$ reflectivity flat 


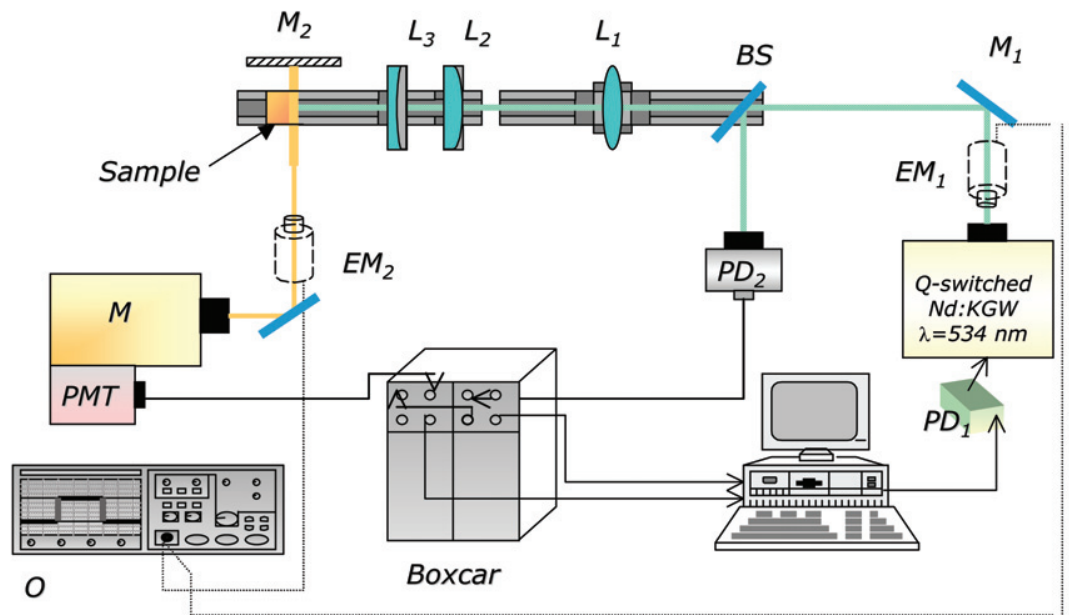

Fig. 6 Schematic of the laser system experimental arrangement. BS: beam splitter; EM: energy meters; $\mathrm{L}_{1}:$ spherical lens; $\mathrm{L}_{2,3}$ : cylindrical lenses; M: monochromator; $\mathrm{M}_{1,2}$ : aluminium flat mirrors; $\mathrm{O}$ : oscilloscope; PD: photodiodes; PMT: photomultiplier tube.

aluminium mirror and the end face of the dye cell or the cylindrical sample as the output coupler, with typical cavity lengths of $2 \mathrm{~cm}$. The pump laser was a frequency-doubled $Q$ switched Nd:KGW (neodymium-doped potassium-gadolinium tungstate: $\left.\mathrm{KGd}\left(\mathrm{WO}_{4}\right)\right)$ laser (Monocrom STR-2+) providing nanosecond pulses with energies of up to $5.5 \mathrm{~mJ}$, beam divergence of $4 \mathrm{mrad}$, and repetition rates of up to $10 \mathrm{~Hz}$. We used a transversal pump scheme, with the exciting pulses being linefocused onto the dye cell or the lateral flat surface of the solid sample by means of a combination of one spherical $(f=50$ $\mathrm{cm})$ and two cylindrical lenses $(f=-15 \mathrm{~cm}$ and $+15 \mathrm{~cm}$, respectively) perpendicularly arranged. Typical pump fluences on the active medium were $180 \mathrm{~mJ} \mathrm{~cm}^{-2}$ in pulses of $10 \mathrm{~ns}$ at $534 \mathrm{~nm}$. Typical beam divergences of the polymeric solid-state dye lasers were $5 \mathrm{mrad}$. Absolute pump and dye laser energies were measured with pyroelectric energy meters. The estimated error in the energy measurements was $10 \%$.

Two notes of caution are needed when analysing the lasing efficiency figures obtained in solid media and comparing them with those obtained in liquid solution. In the first place, the reflectivity of the output coupler of the oscillator cavity has not been optimised for laser operation in any case. Our main concern in our work has been not so much to optimise the laser output as to study the photostability and relative efficiency of operation in different polymeric materials under otherwise identical conditions. Secondly, as indicated above the finishing of the surfaces of the solid samples relevant to laser operation was not laser-grade, which surely impairs their lasing efficiency. Nevertheless, the quality of the finishing was the same for all the solid samples, which allows the lasing efficiency and photostability of all of them to be compared. The reproducibility of the results was checked by performing experiments in which different positions of a given sample or different samples with the same composition were irradiated under the same experimental conditions. In all cases, the results obtained both in efficiency and photostability were the same within the experimental error.

\subsection{Laser operation in liquid solution}

Once the photophysical properties of both commercial and newly synthesized pyrromethene laser dyes dissolved in apolar, polar nonprotic and polar protic solvents were properly characterized, the next step was studying their lasing behaviour in those solvents to find patterns of conduct that could be used as guidelines when trying laser operation in the solid state.

In Table 1 are collected the peak wavelength of the laser emission and the lasing efficiency, defined as the ratio between the laser and pump energies, of commercial dye PM567 in a number of solvents. The photophysical characteristics of PM567 in these same solvents (Section 3.2) were obtained in solutions $2 \times 10^{-6} \mathrm{~mol} \mathrm{l}^{-1}$ (Table 1). However, at this concentration PM567 does not lase, and the lasing characteristics had to be obtained from concentrated dye solutions $\left(1 \times 10^{-3} \mathrm{~mol}\right.$ $\left.1^{-1}\right)$ in which reabsorption/reemission effects are present. A typical spectrum of the laser emission is included in Fig. 1, where it is seen that the lasing bandwidth is much narrower than the fluorescence band.

Good correlations were observed between the fluorescence and lasing properties of PM567 in liquid solutions. ${ }^{41}$ The wavenumbers of fluorescence and lasing maxima were found to have a nearly linear relationship and the lasing efficiency is directly related with the fluorescence quantum yield (Tables 1 and 8): $\operatorname{Eff}(\%)$ values ranging in the $>50,40-50,30-40,<30$ regions approximately correlate with $\phi$ values ranging in the $>0.90,0.85-0.9,0.80-0.85,<0.80$ intervals, respectively. Fig. 7 illustrates the close relationship between lasing efficiency and rate constants of radiative $\left(k_{\mathrm{fl}}=\phi / \tau\right)$ and non-radiative $\left(k_{\mathrm{nr}}=(1-\phi) / \tau\right)$ deactivation processes. It is shown in that figure that the highest lasing efficiencies are obtained in those solvents (polar-protic solvents) where the highest/lowest $k_{\mathrm{ff}} / k_{\mathrm{nr}}$ values, respectively, are observed. Aromatic solvents, on the other hand, exhibited the lowest values in both lasing efficiency and fluorescence yield. These results are in good agreement with the predictions for laser operation based on the photophysical properties of PM567 in liquid solution (Section 3.2).

Dye PM567 is characterized by the presence of ethyl groups at the 2 and 6 positions of the chromophore (Scheme 1). To analyse the effect on photophysical and lasing properties of the molecular structure, non-substituted dye PM546 and dye PM556, with sulfonate groups at the 2 and 6 positions, were also studied. ${ }^{51}$ It was found that, although both their photophysical and lasing properties depend strongly on the 2,6-substituents, the fluorescence properties of these pyrromethene dyes follow the same evolution as their lasing characteristics, not only when a given dye is studied in different solvents (as discussed above) but also when chromophores with different substituents are considered in a common solvent.

Good correlations between the photophysical properties in diluted solutions and the lasing characteristics in moderately concentrated solutions of the novel $\mathrm{P} n \mathrm{AC}(n=1,3,5,10$, and 15) dyes have been observed in different solvents. ${ }^{47}$ The dependence on the nature of the solvent follows the same lines as that exhibited by PM567, with the highest lasing efficiency being obtained in very polar protic solvents, 

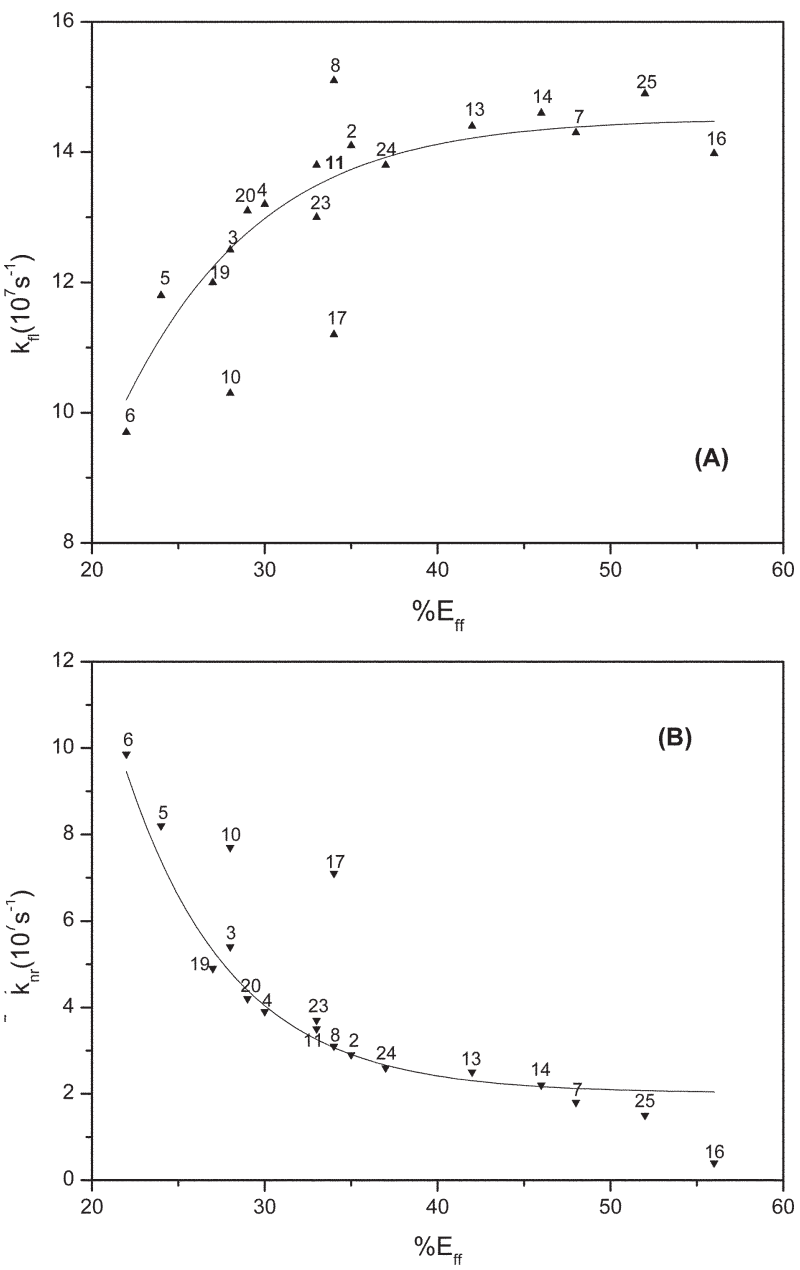

Fig. 7 Relationship between lasing efficiency and the rate constants for the radiative (A) and non-radiative (B) deactivation processes. Solid curves show the tendency of the scattered points. Solvent labels are listed in Table 8 .

such as 2,2,2-trifluoroethanol. In addition, the lasing efficiency increases with the length of the polymethylene chain. However, for the more concentrated solutions needed for optimum laser operation under transversal pumping (optical densities at the excitation wavelength higher than 10 for a 1$\mathrm{cm}$ optical path), the laser efficiency was nearly independent of the nature of the solvent and the length of the polymethylene chain (Table 8)..$^{52}$ The correlation between lasing efficiency and fluorescence quantum yield is preserved in this case, and the $\phi$ values of concentrated $\mathrm{P} n \mathrm{AC}$ (with $n=1,3$, 5 , and 10) solutions become independent of the solvent in polar-aprotic and protic media (Table 4). As discussed in Section 3.4, at the present time we cannot offer any nonspeculative explanation for this effect.

As seen from Tables 8 and 9, all new dyes lase more efficiently than PM567 in ethanol, acetone, ethyl acetate, and cyclohexane, with the dye P10Ac being also more efficient than PM567 in all of the solvents studied. Maximum efficiency was $59 \%$, obtained with a $1.5 \times 10^{-3} \mathrm{~mol}^{-1}$ solution of P10Ac in 2,2,2-trifluoroethanol.

\subsection{Laser operation in solid polymeric matrices}

4.3.1. Pyrromethene 567 dissolved in polymeric media. In a first study, dye PM567 was dissolved into a variety of copolymers of MMA with different monomers such as HEMA, HEA, VP, PEA, and TFMA (Scheme 4) ${ }^{53}$ MMA was chosen as the pivotal component in the formulations developed because the excellent optical transparency and relatively high-laser resistance of PMMA makes this material an obligatory reference in any strategy directed to improve laser performance in polymeric solid-state dye lasers. HEMA, HEA, VP, PEA, and TFMA were chosen with the aim to mimic different solvents of those selected in our previous study of the photophysical and lasing properties of PM567 derivative in liquid solution. The vol/vol proportion of both monomers in each copolymer formulation was systematically varied, and the effect of each composition on the lasing properties of PM567 was evaluated.

In Table 9 are collected relevant laser parameters for solid solutions of PM567 in different copolymers. For comparison, the lasing efficiencies of the same concentration of dye PM567 in liquid solutions using solvents which mimic the above monomers are given in Table 10 (the corresponding lasing efficiencies presented in Table 1 are slightly different in some cases because of the different dye concentration). The data presented in Tables 10 and 11 show that the lasing efficiencies of the solid samples are much lower than those of the liquid solutions in the corresponding solvents. Although poor polishing and thermal lensing effect experienced at the solid-state gain media under our hard excitation regime ${ }^{54}$ could be partly responsible for the differences in efficiency between solid and liquid solutions, it is clear from Table 9 that the composition of the material plays an important role in the effectiveness of the laser operation. The dependence of the lasing efficiency on the matrix composition follows a behaviour similar to that observed in liquid solution, with the best results being obtained in copolymers containing the polar protic monomer TFMA. For a given material the efficiency depended on the relative proportions of the comonomers in the material. We had already observed this behaviour in our studies with dye Rh6G, which was explained in terms of an internal plasticization mechanism. ${ }^{55}$

Table 8 Laser parameters for a number of newly synthesized dipyrromethene $\mathrm{BF}_{2}$ complexes in different solvents. $\lambda_{\mathrm{la}}$ : lasing maximum wavelength; Eff: lasing efficiency in percent. $\mathrm{Nd}: \mathrm{KGW}$ laser (second harmonic) pump energy: $5.5 \mathrm{~mJ}^{\text {pulse }}{ }^{-1}$. Dye concentrations in mol $1^{-1}$ (M). Data taken from ref. 52

\begin{tabular}{|c|c|c|c|c|c|c|c|c|c|c|}
\hline \multirow[b]{2}{*}{ Solvent } & \multicolumn{2}{|c|}{$\mathrm{P} 1 \mathrm{Ac}\left(0.45 \times 10^{-3} \mathrm{M}\right)$} & \multicolumn{2}{|c|}{ P3Ac $\left(1.5 \times 10^{-3} \mathrm{M}\right)$} & \multicolumn{2}{|c|}{ P5Ac $\left(1.5 \times 10^{-3} \mathrm{M}\right)$} & \multicolumn{2}{|c|}{$\mathrm{P} 10 \mathrm{Ac}\left(1.5 \times 10^{-3} \mathrm{M}\right)$} & \multicolumn{2}{|c|}{$\mathrm{P} 15 \mathrm{Ac}\left(0.6 / 2 \times 10^{-3} \mathrm{M}\right)^{a}$} \\
\hline & Eff $(\%)$ & $\lambda_{\mathrm{la}}(\mathrm{nm})$ & Eff $(\%)$ & $\lambda_{\mathrm{la}}(\mathrm{nm})$ & Eff $(\%)$ & $\lambda_{\mathrm{la}}(\mathrm{nm})$ & Eff $(\%)$ & $\lambda_{\text {la }}(\mathrm{nm})$ & Eff $(\%)$ & $\lambda_{\text {la }}(\mathrm{nm})$ \\
\hline 2,2,2-trifluoroethanol & 43 & 577 & 40 & 566 & 48 & 563 & 59 & 563 & 52 & 561 \\
\hline methanol & 43 & 584 & 42 & 565 & 48 & 565 & 55 & 565 & 47 & 562 \\
\hline ethanol & 44 & 579 & 49 & 567 & 50 & 566 & 45 & 566 & 58 & 567 \\
\hline acetone & 47 & 582 & 45 & 565 & 47 & 566 & 45 & 562 & 48 & 564 \\
\hline ethyl acetate & 46 & 580 & 48 & 564 & 51 & 565 & 38 & 566 & 42 & 566 \\
\hline$c$-hexane & 41 & 583 & $36^{b}$ & 568 & 48 & 572 & $c$ & $c$ & 44 & 566 \\
\hline
\end{tabular}

${ }^{a}$ P15Ac concentration was $0.6 \times 10^{-3} \mathrm{~mol}^{-1}$ in 2,2,2-trifluoroethanol, methanol and ethanol, and $2 \times 10^{-3}$ mol $1^{-1}$ in acetone, ethyl acetate and $c$-hexane.

${ }^{b}$ The dye was not completely dissolved even after long periods of sonication. ${ }^{c}$ PB-10-Ac dye did not properly dissolve in $c$-hexane. 
Table 9 Laser parameters ${ }^{a}$ for PM567 dissolved in copolymers of MMA with different monomer compositions. The vol/vol proportion of the comonomers is indicated for each composition. Dye concentration: $1.5 \times 10^{-3}$ M. Nd:KGW laser (second harmonic) pump energy and repetition rate: $5.5 \mathrm{~mJ}$ pulse $^{-1}$ and $1 \mathrm{~Hz}$, respectively. Data taken from ref. 53

\begin{tabular}{|c|c|c|c|c|c|}
\hline \multirow[b]{2}{*}{ Material $^{b}$} & \multirow{2}{*}{$\begin{array}{l}\lambda_{\max } / \\
\mathrm{nm}\end{array}$} & \multirow{2}{*}{$\begin{array}{l}\Delta \lambda / \\
\mathrm{nm}\end{array}$} & \multirow{2}{*}{$\begin{array}{l}\text { Eff } \\
(\%)\end{array}$} & \multicolumn{2}{|c|}{ Laser output $^{c}$} \\
\hline & & & & $I_{5000}(\%)$ & $I_{10000}(\%)$ \\
\hline PMMA & 562 & 7 & 12 & 85 & 70 \\
\hline P(MMA:HEMA 9:1) & 559 & 7 & 7 & 55 & 35 \\
\hline P(MMA:HEMA 8:2) & 560 & 5 & 11 & 50 & 40 \\
\hline P(MMA:HEMA 7:3) & 561 & 5 & 9 & 80 & 70 \\
\hline P(MMA:HEMA 5:5) & 561 & 4 & 10 & 50 & 25 \\
\hline PHEMA & 560 & 4 & 7 & 45 & 10 \\
\hline P(MMA:HEA 9:1) & 560 & 5 & 8 & 40 & 10 \\
\hline P(MMA:HEA 8:2) & 562 & 6 & 11 & 80 & 80 \\
\hline P(MMA:HEA 7:3) & 560 & 5 & 7 & 90 & 80 \\
\hline P(MMA:VP 8:2) & 559 & 5 & 7 & 65 & 40 \\
\hline P(MMA:VP 5:5) & 564 & 6 & 4 & 10 & - \\
\hline P(MMA:PEA 8:2) & 561 & 5 & 7 & 30 & 15 \\
\hline P(MMA:TFMA 9:1) & 563 & 6 & 22 & 75 & 55 \\
\hline P(MMA:TFMA 7:3) & 563 & 6 & 30 & 85 & 60 \\
\hline P(MMA:TFMA 5:5) & 562 & 6 & 23 & 75 & 60 \\
\hline
\end{tabular}

a $\lambda_{\text {max }}$ : peak of the laser emission, $\Delta \lambda$ :FWHM of the laser emission; Eff: energy conversion efficiency. ${ }^{b}$ MMA: methyl methacrylate; HEMA:2-hydroxyethyl methacrylate; HEA: 2-hydroxyethyl acrylate; VP: 1-vinyl-2-pyrrolidone; PEA: 2-phenoxyethyl acrylate; TFMA: 2,2,2-trifluoromethyl methacrylate. ${ }^{c}$ Intensity of the dye laser output after $n$ pump pulses referred to initial intensity $I_{0}, I_{\mathrm{n}}(\%)=100\left(I_{\mathrm{n}} / I_{0}\right)$.

Also collected in Table 9 are data on the evolution of the laser output as a function of the number of pump pulses in the same position of the sample at a repetition rate of $1 \mathrm{~Hz}$. The best photostabilities were obtained with the homopolymer and the 7:3 copolymer formulations. The evolution of the laser output with the number of pump pulses for this materials is shown graphically in Fig. 8 .

Further improvements in both efficiency and photostability of dye PM567could be obtained by optimising the polymer free volume by controlled crosslinking of the polymeric chains. ${ }^{17,56}$ The effect of crosslinking on laser operation was first checked by studying the laser action of PM567 dissolved in solid polymeric matrices of TFMA with different amounts of the crosslinking monomer EGDMA (Scheme 5). ${ }^{38}$ The controlled increase in the rigidity of the host polymer allowed to reach the best compromise between a high concentration of the monomer TFMA and the appropriate hardness of the final material, with the best results both in efficiency and photostability being obtained with the TFMA:EGDMA 90:10 formulation (Fig. 9 and 10). When studying the photophysical properties of these compounds (Section 3.3), it was predicted that the composition TFMA:EGDMA 8:2 was optimum for laser operation. This small discrepancy between the photophysics and lasing results could be due to factors other than those which can affect the lasing properties of the dyes. For instance, the absorbance of the samples at the pump wavelength or the reabsorption and reemission effects for the highly concentrated dye samples used in the lasing experiments, which could be more important in the TFMA:EGDMA 8:2 matrix than in the 9:1 sample due to the higher Stokes shift for the latter system (Table 2).

Once the above approach was demonstrated to work, the next step was to optimise the laser performance by using MMA-based organic materials with increased rigidity, obtained by copolymerisation of MMA with different molar proportions of bi-, tri-, and tetrafunctional methacrylic and acrylic cross-linking monomers such as EGDMA, TEGDA, TMPTMA, PETA, and PETRA (Scheme 5). The copolymerisation of MMA with these monomers leads to a three-dimensional network into which PM567 dye is incorporated. Broadband laser emission, with maxima in the range 559$570 \mathrm{~nm}$, depending on the particular composition of the polymer matrix, and oscillation bandwidths of 4-7 nm, much narrower than the fluorescence spectral widths, were obtained from all the materials under study (Table 11). ${ }^{57}$

From the data in Table 11 is clear that both the proportion and nature of the cross-linking monomer play an important role in the effectiveness of laser operation. With the exception of the monomer EGDMA, the increase of the rigidity of the polymeric matrix by cross-linking improves the lasing efficiency with respect to that exhibited by PM567 embedded in linear PMMA. This laser parameter first increases monotonically with the amount of the cross-linking monomer present in the matrix reaching, for a certain molar proportion of each monomer, an optimum value: $23 \%$ for P(MMA:TMPTMA 98:2); $19 \%$ for P(MMA:TEGDA 90:10); $26 \%$ for P(MMA:PETA 90:10), and 25\% for P(MMA:PETRA 90:10). The initial improvements in efficiency when the concentration of the cross-linking monomer is increased can be attributed to the decrease of the polymer free volume that induces a significant reduction of the rotational and vibrational molecular freedom. ${ }^{17}$ As a result, nonradiative decay of excited dye molecules is prevented leading to a significant increase in the emission quantum yield of the dye. For a certain concentration of the cross-linking monomer, the free volume available within the polymeric matrix will be completely occupied by the dye. Increasing the concentration beyond this point will result in the dye molecules being partially excluded from the shrinking free volume, and formation of dimers and higher aggregates, with their deleterious effect on laser operation, will be forced.

Taking into account the lasing efficiency, it seems that the addition of acrylic monomers to PMMA results in matrices for PM567 dye better than those based on methacrylic ones. Furthermore, to optimise the laser action these latter monomers have to be added in much lower molar proportion $(5 \%$ and $2 \%$ for EGDMA and TMPTMA, respectively) than the acrylic ones (10\% for TEGDA, PETA, and PETRA), which could be related to a slower increase of the glass transition temperature of the matrices based on acrylic monomers. Notwithstanding, the behaviour that both series of monomers induce is similar in that improvements in lasing efficiency are reached as the number of double bonds of each monomer

Table 10 Lasing efficiency (Eff) of PM567 in solvents that mimic the monomers indicated in parenthesis ${ }^{a}$. Dye concentration: $1.5 \times 10-3 \mathrm{M}$. $\mathrm{Nd}: \mathrm{KGW}$ laser (second harmonic) pump energy: $5.5 \mathrm{~mJ}$ pulse $^{-1}$. Data from ref. 53

\begin{tabular}{llllll}
\hline $\begin{array}{l}\text { Solvent } \\
\text { (monomer) }\end{array}$ & $\begin{array}{l}\text { Ethyl acetate } \\
\text { (MMA) }\end{array}$ & $\begin{array}{l}\text { Ethanol } \\
\text { (HEMA; HEA) }\end{array}$ & $\begin{array}{l}\text { 1-methylpyrrolidin- } \\
\text { 2-one (VP) }\end{array}$ & $\begin{array}{l}\text { 2-phenoxyethanol } \\
\text { (PEA) }\end{array}$ & $\begin{array}{l}\text { 2,2,2-trifluoroethanol } \\
\text { (TFMA) }\end{array}$ \\
\hline Eff $(\%)$ & 33 & 37 & 24 & 32 & 52
\end{tabular}

a MMA: methyl methacrylate; HEMA:2-hydroxyethyl methacrylate; HEA: 2-hydroxyethyl acrylate; VP: 1-vinyl-2-pyrrolidone; PEA: 2-phenoxyethyl acrylate; TFMA: 2,2,2-trifluoromethyl methacrylate. 
Table 11 Laser parameters ${ }^{a}$ for PM567 dissolved in MMA crosslinked with different monomers ${ }^{b}$ added in various proportions vol/ vol. Dye concentration: $1.5 \times 10^{-3} \mathrm{M}$. Nd:KGW laser (second harmonic) pump energy: $5.5 \mathrm{~mJ}$ pulse ${ }^{-1}$. Data taken from ref. 57

\begin{tabular}{llll}
\hline Material & $\lambda_{\max }(\mathrm{nm})$ & $\Delta \lambda$ & Eff $(\%)$ \\
\hline P(MMA:EGDMA 95:5) & 566 & 5 & 10 \\
P(MMA:EGDMA 90:10) & 566 & 4 & 9 \\
P(MMA:EGDMA 80:20) & 564 & 7 & 8 \\
P(MMA:TMPTMA 99:1) & 563 & 5 & 21 \\
P(MMA:TMPTMA 98:2) & 563 & 5 & 23 \\
P(MMA:TMPTMA 95:5) & 564 & 5 & 19 \\
P(MMA:TMPTMA 90:10) & 565 & 4 & 14 \\
P(MMA:TMPTMA 80:20) & 561 & 5 & 10 \\
P(MMA:TEGDA 95:5) & 565 & 6 & 14 \\
P(MMA:TEGDA 90:10) & 670 & 6 & 19 \\
P(MMA:TEGDA 80:20) & 566 & 6 & 3 \\
P(MMA:PETA 95:5) & 568 & 5 & 21 \\
P(MMA:PETA 90:10) & 570 & 5 & 26 \\
P(MMA:PETA 80:20) & 569 & 5 & 24 \\
P(MMA:PETRA 99:1) & 559 & 5 & 11 \\
P(MMA:PETRA 98:2) & 562 & 6 & 13 \\
P(MMA:PETRA 95:5) & 564 & 6 & 18 \\
P(MMA:PETRA 90:10) & 563 & 5 & 25 \\
P(MMA:PETRA 80:20) & 563 & 4 & 22
\end{tabular}

${ }^{a} \lambda_{\max }$ : peak of the laser emission, $\Delta \lambda$ : FWHM of the laser emission; Eff: energy conversion efficiency. ${ }^{b}$ MMA: methyl methacrylate; EGDMA: ethylenglycol dimethacrylate; TMPTMA: trimethylolpropane trimethacrylate; TEGDA: tetraethylenglycol diacrylate; PETA: pentaerythritol triacrylate and PETRA: pentaerythritol tetraacrylate.

increases. In fact, the presence of three (TMPTMA and PETA), and four (PETRA) double bonds in lateral chains attached to the same carbon atom (Scheme 5) leads to an overgrown network polymer, which results in the best laser performance of PM567 dye

The stability of the laser output was first studied at the repetition rate of $1 \mathrm{~Hz}$ in matrices of MMA copolymerised with methacrylic comonomer EGDMA and acrylic ones TEGDA and PETA, respectively. ${ }^{57}$ It was found that there is a degree of cross-linking (5\% for EGDMA and PETA, 10\% for TEGDA) that improves the photostability exhibited by PM567 in PMMA homopolymer (Fig. 11). In order to gain a better

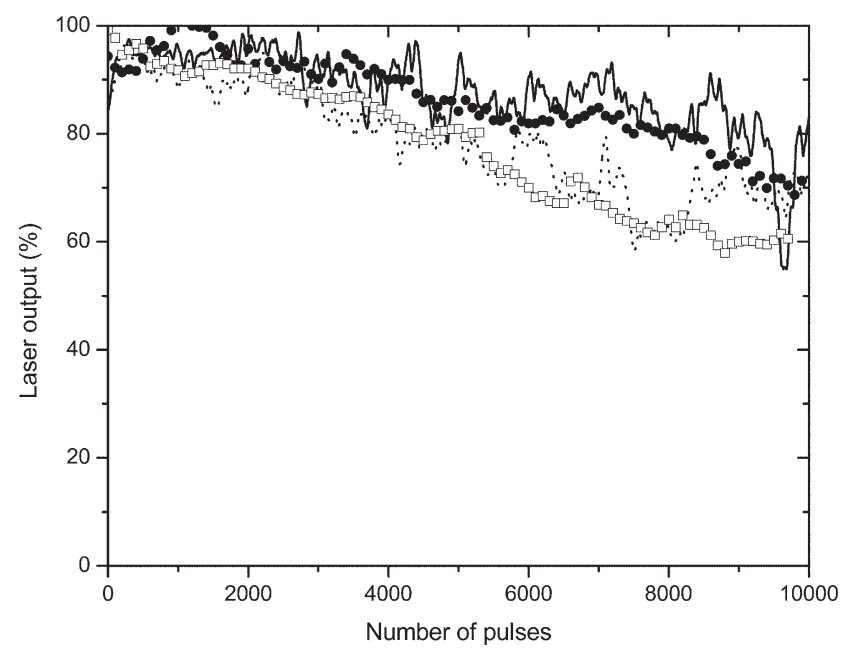

Fig. 8 Normalized laser output as a function of the number of pump pulses for PM567 doped in PMMA homopolymer (full circles), and in different copolymers: P(HEMA:MMA 7:3) (dotted line); P(MMA: HEA 7:3) (solid line), and P(MMA:TFMA 7:3) (open squares). Dye concentration: $1.5 \times 10^{-3} \mathrm{~mol}^{-1}$. Pump energy and repetition rate: $5.5 \mathrm{~mJ}$ pulse ${ }^{-1}$ and $1 \mathrm{~Hz}$, respectively.

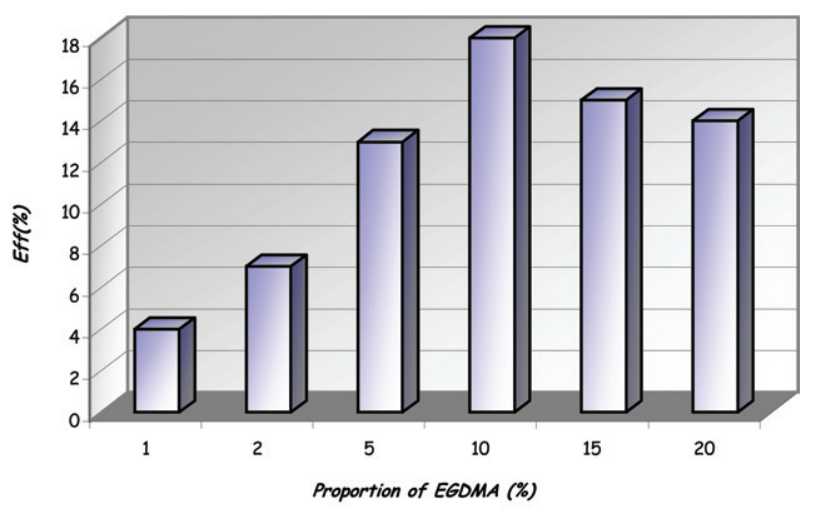

Fig. 9 Dependence on the percentage of the crosslinking monomer EGDMA added to TFMA of the efficiency of the laser emission. Dye concentration: $1.5 \times 10^{-3} \mathrm{~mol} 1^{-1}$. Nd:KGW (second harmonic) pump energy: $5.5 \mathrm{~mJ}$ pulse $^{-1}$.

insight into the photostability of these materials their lifetime was assessed at the more demanding repetition rates of 5 and $10 \mathrm{~Hz}$. In previous studies on dyes of the rhodamine family either dissolved in or covalently bound to methacrylic copolymers, materials were found where the photostability did not change when the repetition rate was increased up to $10 \mathrm{~Hz}$ or $15 \mathrm{~Hz} .^{15,55}$ In the present case, when the pump repetition rate increases also does the degradation rate. Thus, after 10000 pump pulses the emission from the dye dissolved in P(MMA:EGDMA 95:5) and P(MMA:PETA 95:5) dropped to $47 / 26 \%$ and $84 / 50 \%$ of its initial value, respectively, at 5/ $10 \mathrm{~Hz}$ repetition rate. When the matrix was P(MMA:TEGDA 90:10), the laser emission dropped completely after 10000 pulses at $5 \mathrm{~Hz}$ and after only 4000 pulses at $10 \mathrm{~Hz}$. It seems that at high repetition rate the dissipation channels of the energy released to the medium as heat are not fast enough, and as a result the thermal degradation of the dye is enhanced. These results are in good agreement with those obtained in a study on the effect of the heat load on the stability of polymeric dye lasers, where the capability of each material to dissipate the heat generated in the sample as a consequence of the pump energy excitation was characterized by photothermal deflection spectroscopy. ${ }^{58}$ Recent experimental results obtained with this technique ${ }^{59}$ evidenced that the accumulation of heat into the material increased significantly for pumping repetition rates higher than $1 \mathrm{~Hz}$.

As happened with the efficiency, the photostability not only improved by optimising the polymer free volume, but also by

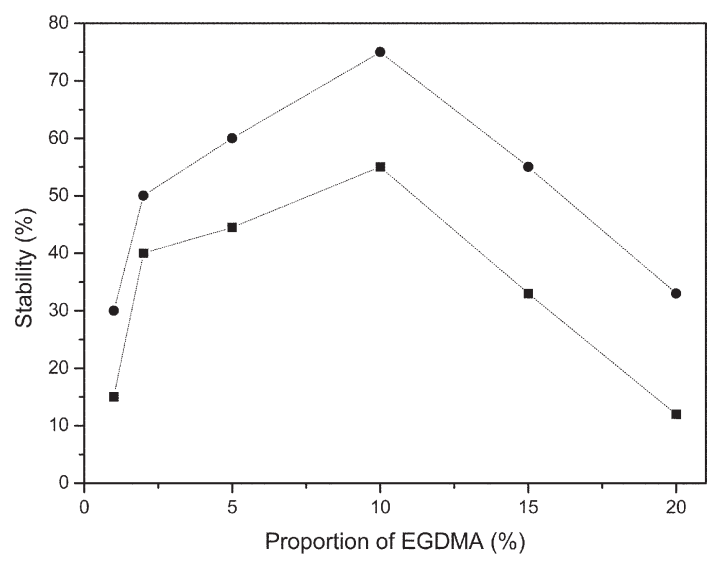

Fig. 10 Dependence on the percentage of the crosslinking monomer EGDMA added to TFMA of the stability of the laser output after $5000(\bullet)$ and 10000 (ם) pump pulses, referred to the initial intensity $I_{0}$ of the laser emission $\left[I_{\mathrm{n}}(\%)=\left(I_{\mathrm{n}} / I_{0}\right) \times 100\right]$. Dye concentration: $1.5 \times 10^{-3} \mathrm{~mol}^{-1}$. Nd:KGW (second harmonic) pump energy and repetition rate: $5.5 \mathrm{~mJ}$ pulse ${ }^{-1}$ and $1 \mathrm{~Hz}$, respectively. 


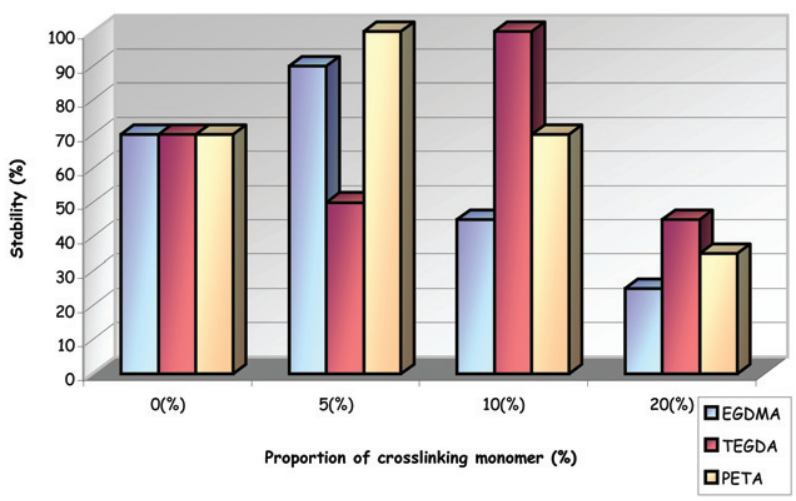

Fig. 11 Dependence on the percentage of the crosslinking monomers EGDMA, TEGDA, and PETA added to MMA of the stability of the laser output after 10000 pump pulses referred to the initial intensity $I_{0}$ of the laser emission $\left[I_{\mathrm{n}}(\%)=\left(I_{\mathrm{n}} / I_{0}\right) \times 100\right]$. Dye concentration: $1.5 \times 10^{-3} \mathrm{~mol}^{-1}$. Nd:KGW (second harmonic) pump energy and repetition rate: $5.5 \mathrm{~mJ}$ pulse $\mathrm{s}^{-1}$ and $1 \mathrm{~Hz}$, respectively.

increasing the number of polymer chains cross-linked by each monomer. Thus, the best performance was reached when the dye was embedded into the matrix P(MMA:PETRA 95:5), where the laser emission remained at $70 \%$ of its initial value after 100000 pump pulses at $5 \mathrm{~Hz}$ repetition rate (Fig. 12). When the repetition rate was $10 \mathrm{~Hz}$, the laser output dropped to $80 \%$ of its initial value after 45000 pump pulses.

4.3.2. Dipyrromethene $\cdot \mathrm{BF}_{2}$ complexes incorporated into PMMA. All the newly synthesized analogs of commercial dye PM567, either dissolved in PMMA (materials PnAC/ PMMA) or copolymerized with MMA (materials COP(PnMA/MMA)) (Scheme 3), exhibited laser action much more efficient and stable than that of dye PM567 dissolved in PMMA (Table 12 and Fig. 13). ${ }^{60}$ Laser emission maxima were in the range 591-594 nm for analogs P1Ac and P1MA and 563-569 for analogs $\mathrm{P} n \mathrm{Ac}$ and $\mathrm{P} n \mathrm{MA}$ with $n=3,5,10$, and 15. Accordingly, analogs P1 present absorption and fluorescence bands placed at lower energies with respect to those of PM567 dye, whereas analogs 3 to 15 return to the original positions of PM567 (Section 3.5 and Table 5).

As seen in Table 12, most of the lasing efficiencies of the modified dyes are in the range $30-40 \%$. This improves significantly our best previous results, obtained with the dye PM567 incorporated into polymeric materials with their composition

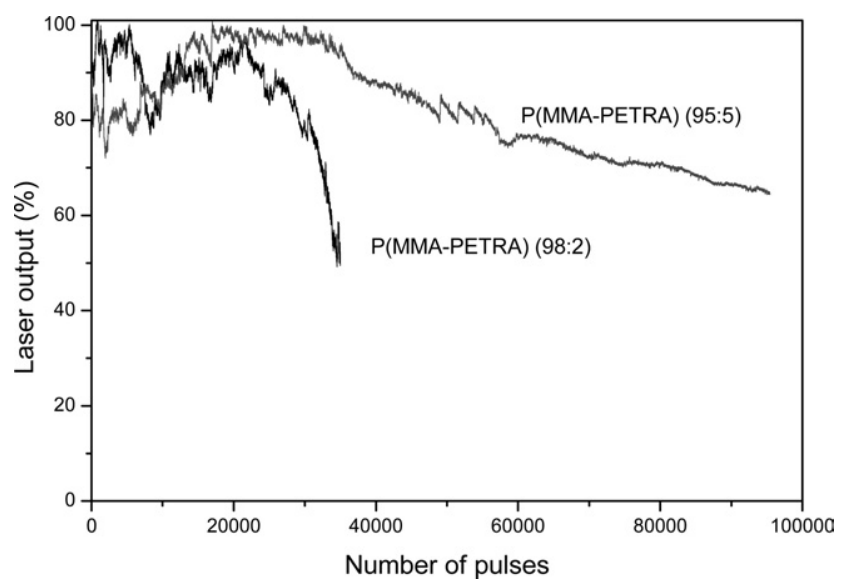

Fig. 12 Normalized laser output as a function of the number of pump pulses for PM567 dissolved in P(MMA:PETRA 98:2) and P(MMA:PETRA 95:5). Dye concentration: $1.5 \times 10^{-3}$ mol $1^{-1}$. $\mathrm{Nd}: \mathrm{KGW}$ (second harmonic) pump energy and repetition rate: 5.5 $\mathrm{mJ}$ pulse $^{-1}$ and $5 \mathrm{~Hz}$, respectively.
Table 12 Laser parameters ${ }^{a}$ for a number of recently synthesized dipyrromethene $\mathrm{BF}_{2}$ complexes either dissolved in PMMA (PnAc/ PMMA) or copolymerized with MMA [COP(PnMA/MMA)]. $\mathrm{Nd}$ KGW laser (second harmonic) pump energy: $5.5 \mathrm{~mJ}$ pulse ${ }^{-1}$. Data taken from ref. 60

\begin{tabular}{lllll}
\hline Material & $c \times 10^{3} / \mathrm{mol}^{-1}$ & $\lambda_{\max } / \mathrm{nm}$ & $\Delta \lambda / \mathrm{nm}$ & Eff $(\%)$ \\
\hline PM567/PMMA & 1.5 & 562 & 7 & 12 \\
P1Ac/PMMA & 0.45 & 591 & 5 & 31 \\
& 1.5 & 591 & 5 & 28 \\
COP(P1MA/MMA) & 0.45 & 593 & 5 & 30 \\
& 1.5 & 594 & 5 & 26 \\
P3Ac/PMMA & 1.5 & 569 & 4 & 36 \\
COP(P3MA/MMA) & 1.5 & 569 & 6 & 34 \\
P5Ac/PMMA & 1.5 & 567 & 4 & 40 \\
COP(P5MA/MMA) & 1.5 & 568 & 5 & 36 \\
P10Ac/PMMA & 1.5 & 566 & 5 & 41 \\
COP(P10MA/MMA) & 1.5 & 564 & 6 & 37 \\
P15Ac/MMA & 0.6 & 564 & 6 & 39 \\
& 1.5 & 563 & 5 & 33 \\
COP(P15MA/MMA) & 0.6 & 565 & 5 & 38 \\
& 1.5 & 564 & 6 & 36
\end{tabular}

${ }^{a} c$ : concentration; $\lambda_{\max }$ : peak wavelength of the laser emission; $\Delta \lambda$ : FWHM of the laser emission; Eff energy conversion efficiency.

optimised for laser operation, where the best efficiencies were in the range $20-30 \%$ (Table 9 ).

The evolution of the laser output as a function of the number of pump pulses in the same position of the sample was studied for the different materials at a repetition rate of $10 \mathrm{~Hz}$ (Fig. 13). It can be appreciated that the photostability of a given modified dipyrromethene $\mathrm{BF}_{2}$ complex is higher when the dye is covalently bonded to the polymeric chains than when the same dye is simply dissolved in the polymer. This is in agreement with the photostability results obtained under continuous irradiation with UV light (Section 3.6). We had observed this same effect in a previous work, ${ }^{15}$ where a modified rhodamine 6G molecule was copolymerized with methacrylic monomers, resulting in a laser material with much higher photostability. We attributed the increase in photostability to the improved dissipation of the excess of absorbed energy: when the dye is covalently bonded to the polymeric chains new channels are open for the elimination of the absorbed pump energy which is not converted into laser emission. Thus, accumulation of heat into the material is prevented. $^{15}$ The most stable materials were copolymers

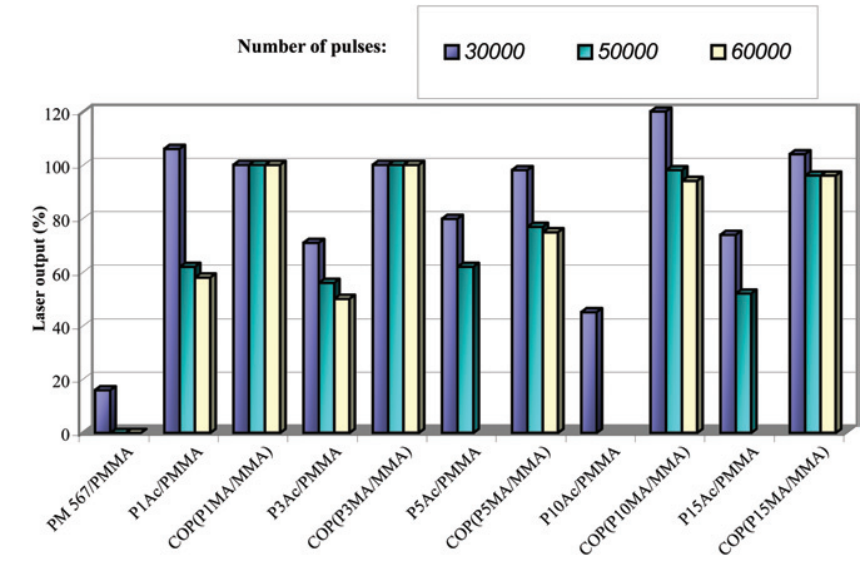

Fig. 13 Percent intensity (referred to initial intensity) of the laser output from the different materials after different numbers of pump pulses in the same position of the sample. Dye concentration: $1.5 \times 10^{-3}$ mol $1^{-1}$. Pump energy and repetition rate: $5.5 \mathrm{~mJ}$ pulse ${ }^{-1}$ and $10 \mathrm{~Hz}$, respectively. 
COP(P1MA/MMA) and COP(P3MA/MMA), which showed no sign of degradation in the laser output after 60000 shots. As an example, in Fig. 14 the evolution of the normalized laser output with the number of pump pulses of copolymer COP(P3MA/MMA) is compared with that of PM567 dissolved in PMMA. These results are an important improvement over the best obtained with dye PM567 incorporated into optimized polymeric materials, reported in the previous section. It is to be expected that by incorporating the newly synthesized pyrromethene $\cdot \mathrm{BF}_{2}$ complexes into polymeric matrices with different degrees of functionalization, using both linear and cross-linking comonomers, further improvements in photostability could be achieved. Work on this line is in progress.

We had observed that in liquid solution analogs P1Ac and P15Ac exhibited higher lasing efficiency at concentrations lower than those used in the present work. ${ }^{47}$ When solid samples with lower concentrations of analogs P1 and P15 were tried as lasing materials, somewhat higher efficiencies were obtained (Table 12), but the photostability was much worst. As discussed in detail in, ${ }^{47}$ the variation of efficiency with dye concentration can be understood in terms of the effect of reabsorption/reemission phenomena on the emission intensity, since the possibility of exciting molecules by absorption of a photon previously emitted by another molecule in the medium depends on the overlapping between absorption and fluorescence spectra, which is affected by the dye concentration. ${ }^{39}$ Thus, lasing efficiency is expected to first increase rapidly with dye concentration until a maximum value is obtained. From this point on, reabsorption/reemission processes hinder further increases in efficiency, and finally result in an efficiency decrease. On the other hand, an increased concentration of the dye can compensate to some extent for the photodegradation of dye molecules caused by the excitation pulses, resulting in an increased photostability.

The results obtained with the modified dipyrromethene $\cdot \mathrm{BF}_{2}$ complexes show a considerably improvement in both laser efficiency and photostability when compared with those obtained with rhodamine dyes under similar experimental conditions. When Rh6G dye was incorporated into a copolymer of HEMA and MMA in the proportion 1:1 vol/vol and pumped with $1.7 \mathrm{~mJ}, 532 \mathrm{~nm}$ pulses from the second harmonic of a Q-switched Nd:YAG laser, laser emission was obtained with an efficiency of $17 \%$ and a lifetime (number of pulses that produces an $80 \%$ drop in the laser output) of 45000 pulses at $10 \mathrm{~Hz}$ repetition rate. ${ }^{55}$

4.3.3. High repetition rate polymeric solid-state dye lasers. Some potential important applications of solid-state dye lasers, such as photodynamic therapy or treatment of portwine stains and other vascular anomalies, would require the laser energy

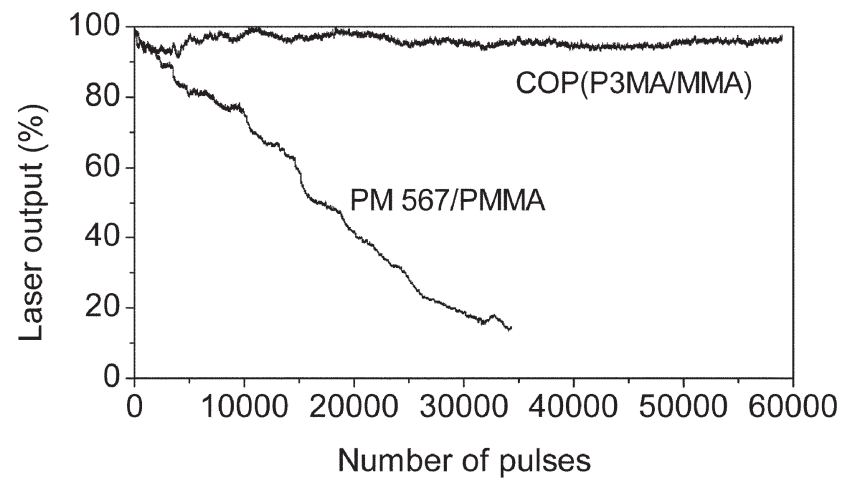

Fig. 14 Normalized laser output as a function of the number of pump pulses for copolymer COP(P3MA/MMA) and for the dye PM567 dissolved in PMMA. Dye concentration: $1.5 \times 10^{-3}$ mol $1^{-1}$. Pump energy and repetition rate: $5.5 \mathrm{~mJ} \mathrm{pulse}^{-1}$ and $10 \mathrm{~Hz}$, respectively. to be applied in high-repetition rate pulses. Except for a work by Bondar et al., ${ }^{61}$ where so-called $F$-type dyes (chemical structure not given) doped in polyurethane acrylate were pumped at $10 \mathrm{kHz}$ rendering laser emission with very low efficiency $(<3.5 \%)$, by the year 2001 all reported work on solid-state dye lasers had been performed at relatively low pulse repetition rates $(<30 \mathrm{~Hz}){ }^{7}$

The absorption band of dye PM567 peaks in the range 515$523 \mathrm{~nm}$, depending on the solvent (Tables 1 and 2), making this dye particularly adequate to be pumped with the $510.6 \mathrm{~nm}$ green line of a high-repetition rate copper-vapor laser. Thus, we prepared solid laser samples in the form of disks, $25 \mathrm{~mm}$ in diameter and $2 \mathrm{~mm}$ thick, of materials PM567/ P(MMA:TFMA 70:30), PM567/P(MMA:TEGDMA 90:10), and PM567/P(MMA:PETA 95:5), which had worked reasonably well at low-repetition rate pumping, and pumped them longitudinally with the green line of a copper-vapor laser at an average power of up to $800 \mathrm{~mW}$ and repetition rate of up to $1 \mathrm{kHz}^{62}$ Samples of $\mathrm{Rh} 6 \mathrm{G}$ dissolved in copolymer P(HEMA:MMA 1:1) were also prepared to be used as reference. The highest efficiency $(37 \%)$ was obtained when the dye was dissolved in P(MMA:PETA 95:5). The evolution of the laser output as a function of time, measured every $5 \mathrm{~min}$, for the different materials is shown in Fig. 15. It is apparent that the good performance of the PM567/P(MMA:PETA 95:5) sample was far superior to that of the other materials. The PM567/P(MMA:PETA 95:5) material produced $290 \mathrm{~mW}$ of initial power at peak wavelength of $550 \mathrm{~nm}$. After an irradiation time of $30 \mathrm{~min}$ (which at $1 \mathrm{kHz}$ corresponds to $1.8 \times 10^{6}$ shots), the output power was still $150 \mathrm{~mW}(52 \%$ of the initial power) and dropped to $32 \mathrm{~mW}$ (11\% of the initial power) after 70 min of operation $\left(4.2 \times 10^{6}\right.$ shots $)$.

Recently, we have demonstrated high repetition rate operation of an all solid-state pulsed dye laser pumped by a diodepumped laser at $10 \mathrm{kHz}{ }^{63}$ The active media investigated were PM567/P(MMA:PETRA 95:5), PM567/P(MMA:PETA 95:5), and Rh6G/P(HEMA:MMA 1:1). Pump radiation at $527 \mathrm{~nm}$ was provided by a frequency-doubled diode-pumped $\mathrm{Nd}$ :YLF laser Q-switched at $10 \mathrm{kHz}$, and pump arrangement was longitudinal. Under a pump power of $3.5 \mathrm{~W}$, initial output power was $560 \mathrm{~mW}$ for Rh6G/P(HEMA:MMA 1:1) (16\% efficiency), $430 \mathrm{~mW}$ for PM567/P(MMA:PETRA 95:5) and 220 $\mathrm{mW}$ for PM567/P(MMA:PETA 95:5), respectively. The evolution of the laser output as a function of time for the different materials is shown in Fig. 16. The best results in this case were obtained with Rh6G/P(HEMA:MMA 1:1), with a decrease in the output to half the initial value after about $6.6 \mathrm{~min}$ (or about 4.0 million shots). In the case of PM567/P(MMA:

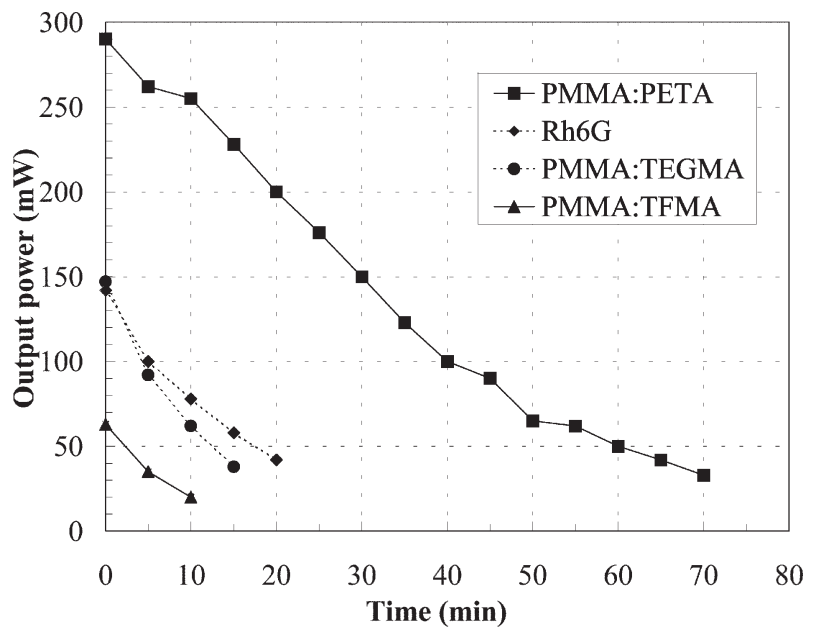

Fig. 15 Evolution of the output power as a function of time for different solid-state dye media pumped with a copper-vapor laser. Pump repetition rate: $1 \mathrm{kHz}$. 


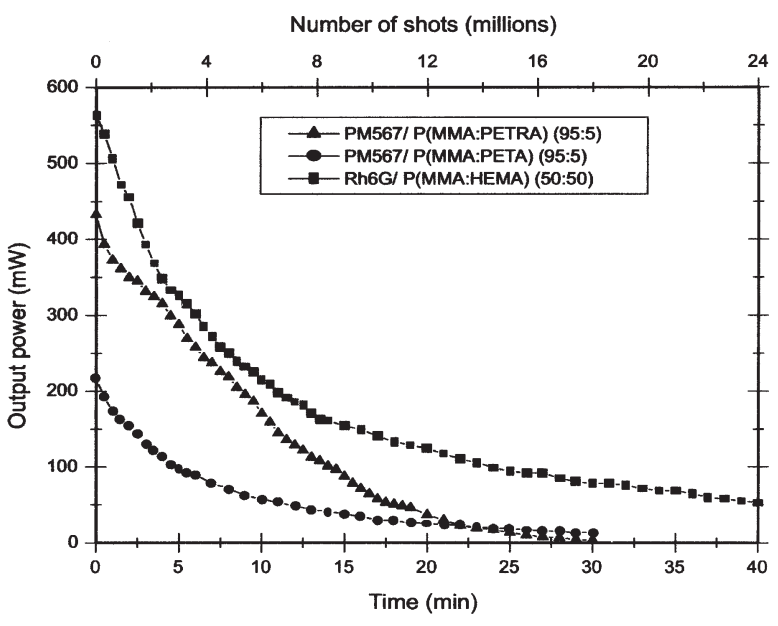

Fig. 16 Evolution of the output power as a function of time for different solid-state dye media pumped with a Nd:YLF (second harmonic) laser. Pump repetition rate: $10 \mathrm{kHz}$.

PETRA 95:5), the output power decreased to half its initial value after about $7.8 \mathrm{~min}$. The wavelengths of the emitted light were about $565 \mathrm{~nm}$ for Rh6G/P(HEMA:MMA 1:1), and about $550 \mathrm{~nm}$ for both PM567/P(MMA:PETRA 95:5) and PM567/P(MMA:PETA 95:5).

4.3.4. Comparison with previous results. For the sake of clarity, a summary of the most representative results previously obtained with pyrromethene $\mathrm{BF}_{2}$ complexes incorporated into polymeric materials is presented in Table 13.

Some care should be taken when comparing our results with the efficiency results collected in Table 13 because our pumping scheme was transversal and the oscillator cavity was not optimised whereas in most of the studies collected in Table 13 pumping was longitudinal and the reflectivity of the output coupler was optimised. In addition, as indicated in section 4.1, the finishing of the surfaces of our solid samples was not laser grade, which surely impairs the efficiency. Even so, in the only study of those collected in Table 13 where a transversal pumping configuration was utilized, the lasing efficiency was not much higher than those obtained with our modified dipyrromethene $\mathrm{BF}_{2}$ complexes (Table 4.6), and this higher efficiency was obtained by using a zigzag optical configuration which increases the optical path of the radiation inside the gain medium.

Direct comparison of our stability results with those obtained by other authors is difficult because of the substantial differences in experimental conditions such as repetition rate or pumping configuration, which can strongly affect the longevity of the solid samples. In order to facilitate comparisons in a way independent of experimental setup, Rahn and King ${ }^{12}$ introduced a normalized photostability defined as the accumulated pump energy absorbed by the system per mole of dye molecules before the output energy falls to one-half its initial value. Its units are gigajoules per mole. Values of this normalized photostability, when available, are included in Table 13 in parenthesis after the corresponding values of the useful lifetime in number of pulses. In our case we can estimate an accumulated absorbed pump energy per mole of dye molecules of $190 \mathrm{GJ} \mathrm{mol}^{-1}$ for PM567/P(MMA:PETRA 95:5) after 100000 pump pulses at $5 \mathrm{~Hz}$ repetition rate, where the laser emission still remained at $70 \%$ of its initial value (Fig. 12). At $10 \mathrm{~Hz}$ repetition rate, the laser emission of materials $\mathrm{COP}(\mathrm{P} 1 \mathrm{MA} / \mathrm{MMA})$ and $\mathrm{COP}(\mathrm{P} 3 \mathrm{MA} / \mathrm{MMA})$ remained at the initial level after an accumulated absorbed pump energy per mole of dye molecules of $114 \mathrm{GJ} / \mathrm{mol}$ (Fig. 13 and 14). Thus, our results compare favourably with those collected in Table 13 and our materials have demonstrated to outperform the commercially available laser dyes.

\section{Conclusions and outlook}

The results presented in this paper, together with those reported in our previous studies on solid state dye-lasers based on rhodamine dyes, indicate that the best dye/host combination is specific for each dye molecule and that there is not an universal polymeric matrix to develop efficient and stable solid-state dye lasers: the photophysical and photochemical properties of each dye require a proper adjustment of the polymer structure composition in order to obtain optical, thermal, and mechanodynamical properties of the matrix which optimise its laser action. On the one hand, the rigidity of the matrix results to be of uppermost importance to optimise the laser performance of a given dye. On the other hand, appropriate chemical modifications in the dye molecules can yield dyes, such as the modified dipyrromethene $\mathrm{BF}_{2}$ complexes described in this work, that lase efficiently and with remarkable photostability when properly incorporated into polymeric matrices, making these solid-state dye lasers fully competitive with their liquid counterparts.

Our research seems to indicate that thermal degradation under laser irradiation of the studied polymeric materials plays an important role in their behaviour, impairing their lasing photostability. As we pointed out in the Introduction section, inorganic glasses have in general better thermal properties than polymers. Thus, one way to improve the thermal resistance of the host material without losing the benefits provided by polymers is using organic-inorganic copolymers, composed of inorganic oxidic structures substituted or cross-linked by organic groups. ${ }^{67-69}$ Our polymer gain media exhibit relatively high negative $\partial n / \partial T$ values, of the order of $-1.4 \times 10^{-4} \mathrm{~K}^{-1}$, ${ }^{70}$ whereas from the literature of crystalline solid-state laser gain media, ${ }^{9}$ we can infer values for $\partial n / \partial T$ of approximately $-0.1 \times 10^{-4} \mathrm{~K}^{-1}$. Thus, organic-inorganic copolymers are expected to exhibit lower $|\partial n / \partial T|$ values than organic polymers with the corresponding decrease in thermal lensing. Previous work ${ }^{54}$ indicates that beam divergence $\Delta \theta$ is a function of the inverse of the focal length of the concave thermal lens. Thus, in organicinorganic copolymers a decrease in $\Delta \theta$ toward its diffraction limit is expected because of the longer focal lengths, or weaker thermal focusing effects.

Organic-inorganic hybrid materials have been already tried as solid hosts for lasing dyes. ${ }^{12,29,71,72}$ Rahn et al. ${ }^{12,29}$ incorporated PM567 into polycom glass, which is a hard and shock-proof glass polymer composite, and obtained under longitudinal pumping lasing efficiencies of $18 \%$ and useful lifetimes of 1200 pulses (corresponding to a normalized photostability of $2.5 \mathrm{GJ} \mathrm{mol}^{-1}$ ) at $1 \mathrm{~Hz}$ repetition rate. ${ }^{12}$ The efficiency reduced to $5 \%$ and the useful lifetime increased to $\sim 4000$ pulses in a deoxygenated matrix. ${ }^{29}$ Yariv et al. ${ }^{71}$ incorporated pyrromethene dyes into composite glasses and organically modified silicate (ORMOSIL) glass samples. Under transversal pumping at $532 \mathrm{~nm}$ they obtained slope efficiencies in the range $23-42 \%$ and useful lifetimes between 11000 and 22000 pump pulses. Recently, Yang et al. ${ }^{72}$ obtained efficiencies of up to $79 \%$ with dye PM567 incorporated into methyltriethoxysilane-derived ORMOSIL under longitudinal pumping, with an output reduction of less than $15 \%$ after 106000 pulses at $2 \mathrm{~Hz}$ repetition rate.

An important problem manifested in these materials is that their optical homogeneity, although clearly superior to that exhibited by porous sol-gel glass, is still inferior to that of polymeric materials ${ }^{11}$ and not high enough for applications requiring good beam quality. It should be possible to control and improve the homogeneity of the hybrid materials through a careful control of the composition, monomer functionality, chemical nature of the organic and inorganic phases, size and morphology of these domains, from nanometer (nanocomposites) to subnanometer (molecular composites) scales, 


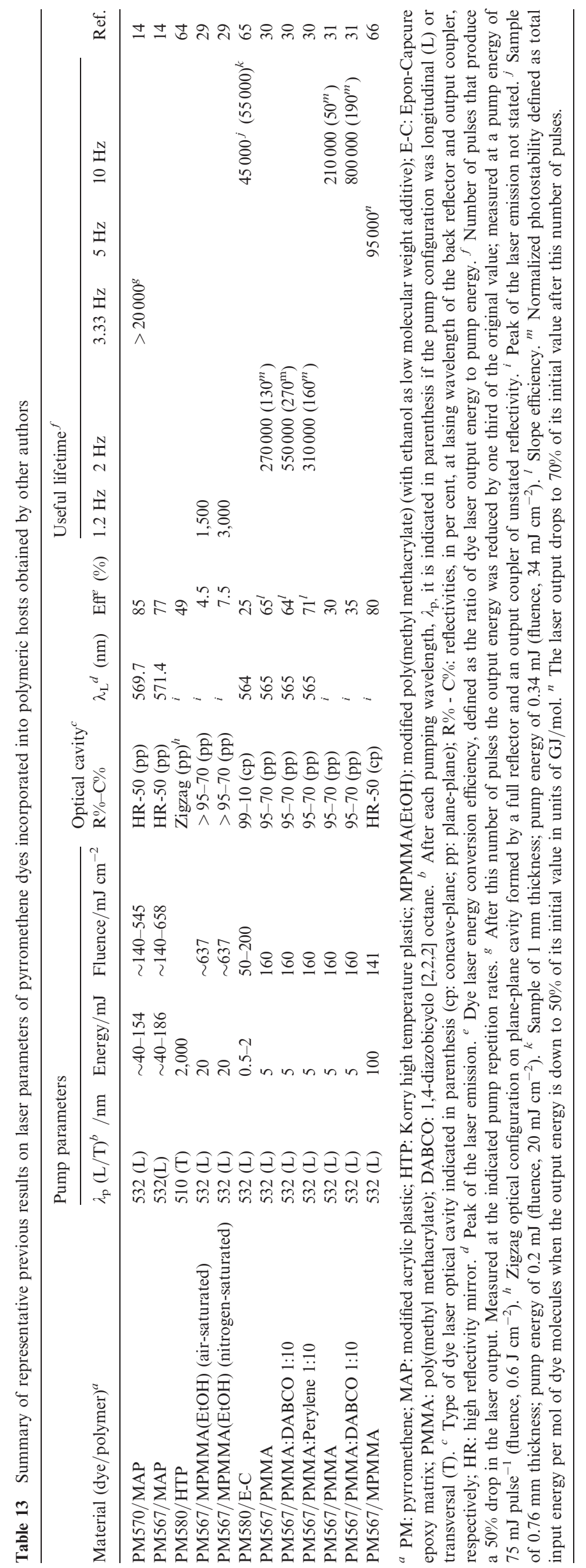


and nature of the interphase interactions. Work on these lines is in progress in our laboratory.

\section{References}

1 Dye Laser Principles, ed. F. J. Duarte and L.W. Hillman, Academic Press, New York,1990.

2 B. H. Soffer and B. B. McFarland, Appl. Phys. Lett., 1967, 10, 266-267.

3 O. G. Peterson and B. B. Snavely, Appl. Phys. Lett., 1968, 12, 238-240.

4 D. A. Gromov, K. M. Dyumaev, A. A. Manenkov, A. P. Maslyukov, G. A. Matyushin, V. S. Nechitailo and A. M. Prokhorov, J. Opt. Soc. Am. B, 1985, 2, 1028-1031.

5 F. Salin, G. Le Saux, P. Georges, A. Brum, C. Bagnall and J. Zarzycky, Opt. Lett., 1989, 14, 785-787.

6 E. T. Knobbe, B. Dunn, P. D. Fuqua and F. Nishida, Appl. Opt., 1990, 29, 2729-2733.

7 A. Costela, I. García-Moreno, and R. Sastre, in Handbook of Advanced Electronic and Photonic Materials and Devices, ed. H. S. Nalwa, Academic Press, San Diego, 2001, vol. 7, p. 161-208.

8 D. N. Nikogosian, in Properties of Optical Laser-Related Materials. A Handbook, John Wiley \& Sons, New York, 1997, p. 388-391.

9 N. P. Barnes, in Tunable Lasers Handbook, ed. F. J. Duarte, Academic Press, New York, 1995, p. 219-291.

10 R. M. O'Connell and T. T. Saito, Opt. Eng., 1983, 22, 393-399.

11 M. D. Rahn and T. A. King, J. Mod. Opt., 1998, 45, 1259-1267.

12 M. D. Rahn and T. A. King, Appl. Opt., 1995, 34, 8260-8271.

13 M. Faloss, M. Canva, P. Georges, A. Brun, F. Chaput and J. P. Boilot, Appl. Opt., 1997, 36, 6760-6763.

14 R. E. Hermes, T. H. Allik, S. Chandra and J. A. Hutchinson, Appl. Phys. Lett., 1993, 63, 877-879.

15 A. Costela, I. García-Moreno, J. M. Figuera, F. Amat-Guerri, R. Mallavia, J. D. Santa-María and R. Sastre, J. Appl. Phys., 1996, 80, 3167-3173.

16 F. J. Duarte, Appl. Opt., 1994, 33, 3857-3860.

17 R. Sastre and A. Costela, Adv. Mater., 1995, 7, 198-202.

18 A. Costela, I. García-Moreno, J. M. Figuera, F. Amat-Guerri and R. Sastre, Laser Chem., 1998, 18, 63-84.

19 T. G. Pavlopoulos, M. Shah and J. H. Boyer, Appl. Opt., 1988, 27, 4998-4999.

20 T. G. Pavlopoulos, M. Shah and J. H. Boyer, Opt. Commun., 1989, 70, 425-427.

21 M. Shah, K. Thangaraj, M.-L. Soong, L. T. Wolford, J. H. Boyer, I. R. Politzer and T. G. Pavlopoulos, Heteroat. Chem., 1990, 1, 389-399.

22 T. G. Pavlopoulos, J. H. Boyer, M. Shah, K. Thangaraj and M.-L. Soong, Appl. Opt., 1990, 29, 3885-3886.

23 T. G. Pavlopoulos, J. H. Boyer, K. Thangaraj, G. Sathyamoorthi, M. P. Shah and M.-L. Soong, Appl. Opt., 1992, 31, 7089-7094.

24 S. C. Guggenheimer, J. H. Boyer, K. Thangaraj, M. P. Shah, M.-L. Soong and T. G. Pavlopoulos, Appl. Opt., 1993, 32, 3942-2943.

25 J. H. Boyer, A. M. Haag, G. Sathyamoorthi, M.-L. Soong, K. Thangaraj and T. G. Pavlopoulos, Heteroat. Chem., 1993, 4, 39-49.

26 M. P. O’Neil, Opt. Lett., 1993, 18, 37-38.

27 W. P. Partridge, Jr., N. M. Laurendeau, C. C. Johnson and R. N. Steppel, Opt. Lett., 1994, 19, 1630-1632.

28 Y. Assov, Z. Burshtein and S. Rosenwaks, Appl. Opt., 1998, 37, 4914-4920.

29 M. D. Rahn, T. A. King, A. Gorman and I. Hamblett, Appl. Opt., 1997, 36, 5862-5871.

30 M. Ahmad, M. D. Rahn and T. A. King, Appl. Opt., 1999, 38, 6337-6342.

31 M. Ahmad, T. A. King, D. Ko, B. H. Cha and J. Lee, Opt. Commun., 2002, 203, 327-334.

32 E. Vos de Wael, J. A. Pardoen, J. A. van Koeveringe and J. Lugtenburg, J. R. Neth. Chem. Soc., 1977, 96, 306-309.

33 H. J. Wories, J. H. Koek, G. Lodder, J. Lugtenburg, R. Fokkens, O. Driesen and G. R. Mohn, Recl. Trav. Chim. Pays-Bas., 1985, 104, 288-291.

34 F. Amat-Guerri, M. Carrascoso, M. Liras and R. Sastre, Photochem. Photobiol., 2003, 77, 577-584.

35 These laser-emitting copolymers are presently covered by $\mathrm{F}$. Amat-Guerri, M. L. Carrascoso, A. Costela, M. Rodríguez, R. Sastre and I. García-Moreno, Span. Pat. ES9901540, 1999.
36 N. B. Delone, in Interaction of Laser Radiation with Matter, Nauka, Moscow, 1989, p. 280

37 T. López Arbeloa, F. López Arbeloa, P. Hernández Bartolome and I. López Arbeloa, Chem. Phys., 1992, 160, 123-130.

38 A. Costela, I. García-Moreno, R. Sastre, F. López Arbeloa, T. López Arbeloa and I. López Arbeloa, Appl. Phys. B, 2001, 73, 19-24.

39 I. López Arbeloa, J. Photochem., 1980, 14, 97-105.

40 A. Bergmann, W. Holzer, R. Stark, H. Gratz, A. Penzkofer, F. Amat-Guerri, A. Costela, I. Garcia-Moreno and R. Sastre, Chem. Phys., 2001, 271, 201-213.

41 F. López Arbeloa, T. López Arbeloa, I. López Arbeloa, I. García-Moreno, A. Costela, R. Sastre and F. Amat-Guerri, Chem. Phys., 1998, 236, 331-341.

42 F. López Arbeloa, P. Ruiz Ojeda and I. López Arbeloa, J. Photochem. Photobiol. A, 1988, 45, 313-323.

43 F. López Arbeloa, A. Costela and I. López Arbeloa, J. Photochem. Photobiol. A, 1990, 55, 97-103.

44 J. B. Birks in Photophysics of Aromatic Molecules, WileyInterscience, London, 1970.

45 F. López Arbeloa, T. López Arbeloa, I. López Arbeloa, A. Costela, I. García-Moreno, J. M. Figuera, F. Amat-Guerri and R. Sastre, Appl. Phys. B, 1997, 64, 651-657.

46 S. J. Strickler and R. A. Berg, J. Chem. Phys., 1962, 37, 814-822.

47 A. Costela, I. García-Moreno, C. Gómez, R. Sastre, F. AmatGuerri, M. Liras, F. López Arbeloa, J. Bañuelos Prieto and I. López Arbeloa, J. Phys. Chem. A, 2002, 106, 7736-7742.

48 F. López Arbeloa, J. Bañuelos Prieto, I. López Arbeloa, A. Costela, I. García-Moreno, C. Gómez, F. Amat-Guerri, M. Liras and R. Sastre, Photochem. Photobiol., 2003, 78, 30-36.

$49 \mathrm{Ph}$. Herbert, G. Baldacchino, Th. Gustavsson and J. C. Mialocq, J. Photochem. Photobiol. A: Chem., 1994, 84, 45-55.

50 W. Holzer, H. Gratz, T. Schmitt, A. Penkofer, A. Costela, I. García-Moreno, R. Sastre and F. J. Duarte, Chem. Phys., 2000, 256, 125-136.

51 F. López Arbeloa, T. López Arbeloa, I. López Arbeloa, I. GarcíaMoreno, A. Costela, R. Sastre and F. Amat-Guerri, Chem. Phys. Lett., 1999, 299, 315-321.

52 A. Costela, I. García-Moreno, C. Gómez, F. Amat-Guerri and R. Sastre, Appl. Phys. Lett., 2001, 79, 305-307.

53 A. Costela, I. García-Moreno, J. Barroso and R. Sastre, Appl. Phys. B, 2000, 70, 367-373.

54 F. J. Duarte, A. Costela, I. García-Moreno, R. Sastre, J. J. Ehrlich and T. S. Taylor, Opt. Quantum Electron., 1997, 29, 461-472.

55 A. Costela, F. Florido, I. García-Moreno, R. Duchowicz, F. Amat-Guerri, J. M. Figuera and R. Sastre, Appl. Phys. B, 1995, 60, 383-389.

56 A. Costela, I. García-Moreno, J. Barroso and R. Sastre, Appl. Phys. B, 1998, 67, 167-173.

57 A. Costela, I. García-Moreno, C. Gómez, O. García and R. Sastre, J. Appl. Phys., 2001, 90, 3159-3166.

58 R. Duchowicz, L. B. Scaffardi, A. Costela, I. García-Moreno, R. Sastre and A. U. Acuña, Appl. Opt., 2000, 39, 4959-4963.

59 R. Duchowicz, L. B. Scaffardi, A. Costela, I. García-Moreno, R. Sastre and A. U. Acuña, Appl. Opt., 2003, 42, 1029-1035.

60 A. Costela, I. García-Moreno, C. Gómez, F. Amat-Guerri, M. Liras and R. Sastre, Appl. Phys. B, 2003, 76, 365-369.

61 M. V. Bondar, O. V. Przhonskaya, A. G. Romanov, E. A. Tikhonov and ans A. F. Khomenko, Sov. Phys. Tech. Phys., 1986, 31, 1439

62 A. Costela, I. García-Moreno, R. Sastre, D. W. Coutts and C. E. Webb, Appl. Phys. Lett., 2001, 79, 452-454.

63 K. M. Abedin, M. Alvarez, A. Costela, I. García-Moreno, O García, R. Sastre, D. W. Coutts and C. E. Webb, Opt. Commun., 2003, 218, 359-363.

64 A. Mandl, A. Zavriyev and D. E. Klimek, IEEE J. Quantum Electron., 1996, 32, 1723-1726.

65 M. J. Cazeca, X. L. Liang, J. Kumar and S. K. Tripathy, Appl. Opt., 1997, 36, 4965-4968.

66 D. Pacheco, H. R. Aldag, in Solid-State Lasers VII (Proc. SPIE), ed. R. Scheps, 1998, 3265, 2-12.

67 B. M. Novak, Adv. Mater., 1993, 6, 422-433.

68 C. Sánchez and F. Ribot, New J. Chem., 1994, 18, 1007-1047.

69 U. Schubert, N. Hüsing and A. Lorentz, Chem. Mater., 1995, 7, 2010-2027.

70 F. J. Duarte, A. Costela, I. García-Moreno and R. Sastre, Appl. Opt., 39, 6522-6523.

71 E. Yariv and R. Reisfeld, Opt. Mater., 1999, 13, 49-54.

72 Y. Yang, G. Qian, Z. Wang and M. Wang, Opt. Commun., 2002, 204, 277-282. 